\title{
Critical and umbilical points of a non-Gaussian random field
}

\author{
T. H. Beuman, ${ }^{1}$ A. M. Turner, ${ }^{2}$ and V. Vitelli ${ }^{1, *}$ \\ ${ }^{1}$ Instituut-Lorentz for Theoretical Physics, Leiden University, NL 2333 CA Leiden, The Netherlands \\ ${ }^{2}$ Institute for Theoretical Physics, Universiteit van Amsterdam, NL 1090 GL Amsterdam, The Netherlands
}

(Received 13 November 2012; revised manuscript received 10 April 2013; published 15 July 2013)

\begin{abstract}
Random fields in nature often have, to a good approximation, Gaussian characteristics. For such fields, the number of maxima and minima are the same. Furthermore, the relative densities of umbilical points, topological defects which can be classified into three types, have certain fixed values. Phenomena described by nonlinear laws can, however, give rise to a non-Gaussian contribution, causing a deviation from these universal values. We consider a random surface, whose height is given by a nonlinear function of a Gaussian field. We find that, as a result of the non-Gaussianity, the density of maxima and minima no longer match and we calculate the relative imbalance between the two. We also calculate the change in the relative density of umbilics. This allows us not only to detect a perturbation, but to determine its size as well. This geometric approach offers an independent way of detecting non-Gaussianity, which even works in cases where the field itself can not be probed directly.
\end{abstract}

DOI: 10.1103/PhysRevE.88.012115

PACS number(s): 02.50.Ey, 02.40.Xx, 02.40.Pc, 02.40.Ky

A wide range of phenomena feature observables that can be regarded as random fields. The cosmic background radiation [1] is a famous example, but the height profile of a growing surface [2], medical images of brain activity [3], and optical speckle patterns $[4,5]$ also demonstrate this.

In many cases, the fields can be approximated as Gaussian fields, meaning that they have certain properties that are related to the Gaussian (or normal) distribution. This is, for example, the case when the observable signal is averaged over a large scale, producing approximately Gaussian statistics on account of the central limit theorem. The stochastic properties of such fields have already been the subject of several studies [5-10]: the density of maxima and minima for instance reflects the amount of field fluctuations at short distances.

Analytical investigations are often restricted to such Gaussian fields. However, phenomena described by nonlinear laws produce non-Gaussian signals. Since these nonlinear effects are usually quite small, the resulting departures from Gaussianity can be tiny. Nevertheless, these non-Gaussianities can offer a key to understanding the interesting nonlinear processes behind the phenomena in question.

If the non-Gaussianity is generated by microscopic nonlinear processes, then some indicator that is sensitive to short distances would be necessary to observe it. Microscopic dynamics do not involve mixing between different regions [11,12], so the originally Gaussian field $H(\vec{r})$ simply transforms in a local way, $H(\vec{r}) \rightarrow F_{N L}[H(\vec{r})]$. Provided that this transformation is nonlinear, the new function will have non-Gaussian statistics.

The standard approach to describing the statistics of a random field is to measure its correlation functions. In the case of a two-dimensional random scalar field $h(x, y)$ with Gaussian statistics, its statistical properties are entirely encoded in its two-point correlation function $\left\langle h(x, y) h\left(x^{\prime}, y^{\prime}\right)\right\rangle$ [as a function of the distance between $(x, y)$ and $\left.\left(x^{\prime}, y^{\prime}\right)\right]$. The higherorder correlation functions can be factorized into two-point correlation functions, by Wick's theorem. A breakdown in these relationships is evidence that the field is not Gaussian.

*vitelli@lorentz.leidenuniv.nl
In this paper, we take a geometric approach to tackle this problem. We interpret the scalar field as the height of a surface (see Fig. 1) and infer the statistical properties of the signal by studying the stochastic topography of this surface [13]. Such an approach has already been the subject of both theoretical [6,7,14-16] and experimental studies [4].

First, we focus on the statistical imbalance between peaks and troughs. A test of Gaussianity based on similar ideas has already been applied to the temperature fluctuations in the cosmic microwave background $[17,18]$.

We will focus on the difference between the densities of maxima and minima. This should also be sensitive to local statistics of the field, but it will be a measurement of the non-Gaussian properties in particular since a Gaussian variable is always symmetric around its mean value. We will study signals of the form $F_{N L}(H)$ where the underlying field $H$ is Gaussian and $F_{N L}$ is any nonlinear function, and we will find that the imbalance can be nonzero, illustrating this approach. Moreover, we show how large the imbalance is exactly in relation to the nonlinear perturbation, which allows one to attack the reverse problem: by measuring the difference in density between maxima and minima for a given near-Gaussian field, one can quantify the size of the non-Gaussian component.

Next, we turn to a class of singular points of the surface, known as umbilics, that do not depend on how the surface is oriented in space. In order to understand the geometrical meaning of umbilical points, imagine drawing at every point on the surface the two principal directions, along which its curvature is maximal or minimal. At some locations, the principal directions can not be defined because the curvature is the same along all directions: these special points are called umbilics. As we shall see, umbilical points are topological defects with an index of $\pm \frac{1}{2}$.

This geometrical construct is very useful in a number of physical contexts. In statistical optics, the surface may represent a curved wavefront that emerges when a plane wave is passed through an inhomogeneous refracting medium. In this mapping, the normals to the surface are light rays and the umbilical points correspond to the regions where the wave attains its maximal intensity. In two-dimensional elasticity 


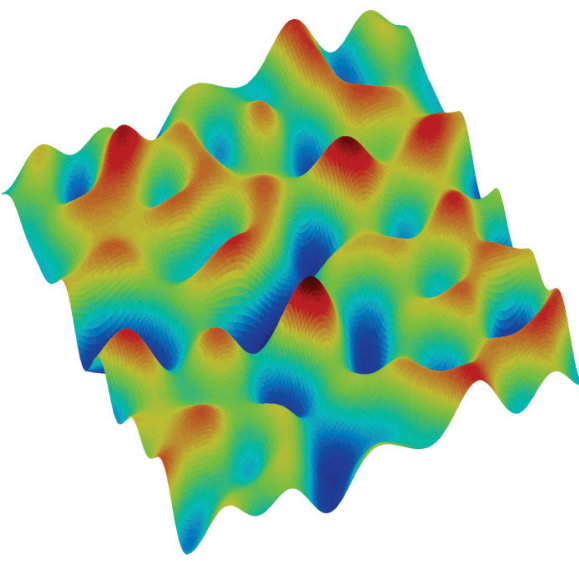

FIG. 1. (Color online) A realization of a Gaussian field with periodic boundary conditions.

or fluid flow, the surface can represent a potential function of two variables, the second derivatives from which a shear field can be defined that corresponds to the principal curvature directions of the surface. The points where the shear field vanishes correspond to the umbilical points.

The umbilical points of a surface can be classified into three types: lemons, monstars, and stars. A striking statistical feature of surfaces whose height fluctuates spatially like an isotropic Gaussian random field is that the densities of the three types of umbilics have fixed ratios, which are universal numbers $[7,16]$. This property can therefore be used to test whether a given isotropic field is Gaussian; if for a given field $h$ the relative densities are found to differ from the universal values, one may immediately conclude that the field under consideration is not an isotropic Gaussian one. Crucially, such a test requires only that the line field corresponding to the principal curvature directions is measurable: the statistics of the scalar height field from which the curvature directions are derived can be probed without being directly observed.

To give an example of a case where the near-Gaussian field of interest is not directly observable, consider the phenomenon of weak gravitational lensing [19]. As stipulated by the theory of general relativity, matter bends space-time, which also affects light rays. The light from a distant galaxy, for instance, does not come to us in a straight line due to the presence of matter between that galaxy and us. As a result, we see a distorted image of the galaxy. In general, a circular object will look like an ellipse. While most of the matter in the universe is believed to be made up of dark matter which we can not (yet) detect, the shear field can be detected. The near-Gaussian field in this case is obtained by projecting the mass onto the sky, along the lines of sight. This is called the projected gravitational potential. On large scales, this field is approximately Gaussian by virtue of the central limit theorem since the projection involves summing over a lot of regions that are randomly distributed. On smaller scales, however, interactions can give rise to non-Gaussian contributions. If we interpret the projected gravitational potential as a (nearGaussian) surface, then the shear direction corresponds to the principal direction of this surface [20]. In terms of the shear field, they correspond to points in the sky where a circular light source still appears circular.
Another example of a physical process in which umbilical points can prove their usefulness is in the context of optical speckle fields. These fields arise, for example, when a coherent beam of light scatters from a rough surface. Since the many reflected waves become superimposed, this produces a random pattern of intensity with approximately Gaussian statistics. In this case, it is the points of circular polarization that can be identified as umbilical points. The relative densities of the various types of umbilical points have been found to match the theoretical predictions in experiments [4]. A speckle field is not always Gaussian. First, when the surface is not that rough, the superposition of the reflected waves will not be sufficiently random. Second, a light beam could be transmitted through a random medium to map out the statistics of its index of refraction.

Other contexts in which umbilical points can offer a window for non-Gaussianity include polarization singularities in the cosmic microwave background [21-24], topological defects in a nematic [25,26], and a superfluid near criticality [27,28].

Testing whether the three types of umbilical points occur in their prescribed ratios can thus reveal whether a non-Gaussian component is present in a given field. However, it does not provide any quantitative information on the size of the nonGaussianity. In this paper, we address precisely this issue by calculating how much the relative densities of umbilical points deviate from the universal values in relation to the type and size of the perturbation. Aside from being applicable even when the field itself can not be observed directly, the approach based on umbilics provides an additional probe, should the extrema test not be sensitive enough. As an illustration, consider the case $h(\vec{r})=H(\vec{r})+\varepsilon H(\vec{r})^{3}$, where $H(\vec{r})$ is a Gaussian field. Since the perturbation is an odd function of $H$, the symmetry between positive and negative values of $H$ is preserved and the densities of maxima and minima will not differ. By contrast, a study of the umbilical points does reveal the non-Gaussianity of $h$, as we will show.

The outline of this paper is as follows. In Sec. I, we review the properties of Gaussian fields and introduce the basic notions and notations that we will use. We then demonstrate how the imbalance between maxima and minima can be calculated in Sec. II. In the process, we determine the probability distribution for the values of minima in a Gaussian field. The final result is compared with results from computer generated fields. In Sec. III, we introduce the necessary geometric concepts concerning umbilical points and proceed to determine how the fraction of monstars deviates from the universal value for Gaussian fields in relation to the applied perturbation. The result is again compared to results from computer simulations. Finally, Sec. IV provides a summary and conclusions.

\section{GAUSSIAN FIELDS}

The Gaussian distribution is the archetype of a continuous probability density. It is given by

$$
f(x)=\frac{1}{\sqrt{2 \pi} \sigma} \exp \left[-\frac{1}{2}\left(\frac{x-\mu}{\sigma}\right)^{2}\right],
$$

where $\mu$ and $\sigma$ are the expectation value and standard deviation of the stochastic variable, respectively. One of its special 
properties is that the sum of two independent stochastic variables, which adhere to this distribution, is itself also a Gaussian variable, albeit of course with $\mu=\mu_{1}+\mu_{2}$ and $\sigma^{2}=\sigma_{1}^{2}+\sigma_{2}^{2}$. This property can be considered to be one of the components of the proof of the central limit theorem, which states that, under some very general conditions, the sum (or average) of a large number of independent stochastic variables acquires a Gaussian distribution, in the limit that the number goes to infinity [29]. Because of this, many random processes can be well approximated using a Gaussian distribution, e.g., the number of times a (fair) coin comes up heads when it is flipped a (large) number of times, or the amount of rain that falls at a certain spot during a year.

A Gaussian random field is an extension of this principle to two dimensions. For instance, one might consider the amount of rain that falls at different places throughout an area rather than a single spot. Upon adding together all the contributions of all rain clouds during the course of a year, one obtains a random field.

Formally, a field is a stochastic function $H(\vec{r})$. The minimum requirement for a Gaussian field is that the probability distribution of $H\left(\vec{r}_{0}\right)$ at any point $\vec{r}_{0}$ has to be described by a Gaussian. More generally, if we consider the values that the field attains at any number of points, $\xi_{1}=H\left(\vec{r}_{1}\right), \xi_{2}=$ $H\left(\vec{r}_{2}\right), \ldots, \xi_{n}=H\left(\vec{r}_{n}\right)$, the joint probability distribution has to be of the form

$$
p\left(\xi_{1}, \ldots, \xi_{n}\right) \propto \exp \left(-\frac{1}{2} \sum_{i, j} A_{i j} \xi_{i} \xi_{j}\right),
$$

where $A_{i j}$ are constants. These constants give information about the relative values at different points (which would be useful, for example, if we wanted to know the distribution of the derivative of the field).

Any well-behaved Gaussian field can be decomposed into Fourier modes, resulting in the sum of an infinite number of wave functions

$$
\psi(\vec{r})=\psi_{0}+\sum_{\vec{k}} A(\vec{k}) \cos \left(\vec{k} \cdot \vec{r}+\phi_{\vec{k}}\right) .
$$

This shows how much of the fluctuations occur at each wavelength, for example, a surface of water might fluctuate with some random waves. If that is due to some external sound at a certain frequency, the Fourier transform will be strongest at the corresponding wavelength.

This procedure may also be turned around; a Gaussian field may be generated by summing up a large number of Fourier modes. We will now discuss a field that is generated in this way and try to understand how the statistics of the phase factors $\phi_{\vec{k}}$ reflect properties of the field, such as Gaussianity and translational invariance.

The defining characteristic of a Gaussian field is now that the phases $\phi_{\vec{k}}$ are random and completely uncorrelated to each other. Already, by translational invariance, second order correlations between $\phi_{k}$ and $\phi_{k^{\prime}}$ are ruled out. If the phases are completely independent, then at each individual point $\vec{r}, \psi(\vec{r})$ is the sum of an infinite number of independent random numbers between -1 and 1 (as a result of the cosine), each weighted with a factor $A(\vec{k})$. Thus, from the central limit theorem $\psi(\vec{r})$ is a Gaussian random variable. In contrast, in a non-Gaussian field the phases are correlated, i.e., the phases of different modes depend on each other. This mechanism is often called mode coupling.

So far, no statements have been made about the function $A(\vec{k})$ : it has no influence on the Gaussianity (nor on the homogeneity) of $\psi$. Indeed, this function is a free parameter, called the amplitude spectrum. While all Gaussian fields share some general properties, other more specific properties (such as the density of critical points, as we shall see) depend on this amplitude spectrum. For example, when $A(\vec{k})$ is large for vectors $\vec{k}$ with a small norm, the field $\psi$ is dominated by these waves with small wave vectors and hence large wavelengths, resulting in a more slowly varying $\psi$ as compared to a Gaussian field that is dominated by large wave vectors.

There is one more condition that we will pose: next to being homogeneous, we will also only consider fields that are isotropic, i.e., have rotational symmetry. This is achieved by requiring that $A(\vec{k})$ depends on the magnitude of $\vec{k}$ only, i.e., $A(\vec{k})=A(k)$.

In order to make a clear distinction between Gaussian and non-Gaussian, we will use $H$ to indicate an (isotropic) Gaussian field and $\psi$ for any (homogeneous and isotropic) field. Later, we will also use $h$ to indicate a perturbed Gaussian field.

When we have a Gaussian variable $x$ with a certain $\mu$ and $\sigma$, we can make a transformation to $y=\frac{x-\mu}{\sigma}$, which is then a standard Gaussian variable, having $\mu=0$ and $\sigma=1$. This translation and rescaling has no effect on the overall properties of $x$ and is introduced for convenience. We will apply a similar transformation by setting $\langle H\rangle=0$ and $\left\langle H^{2}\right\rangle=1$. The expectation values are obtained by integrating over all possible values of all random variables, which in this case, are the uniformly distributed phases:

$$
\langle\ldots\rangle \equiv\left(\prod_{\vec{k}} \int \frac{d \phi_{\vec{k}}}{2 \pi}\right) \ldots
$$

For our earlier definition (3), the normalization translates to $H_{0}=0$ and $\sum_{\vec{k}} \frac{1}{2} A(k)^{2}=1$. This normalization is for the purpose of simplicity only and has no impact on our analysis.

More details on these calculations, as well as additional properties of Gaussian fields and definitions, can be found in Appendix A. There we also demonstrate how the two-point correlation function can be derived from Eq. (3). We also show how the higher-order correlation functions are related to the two-point ones. Testing whether these relations hold for a given field $\psi$ can reveal whether $\psi$ is Gaussian or not. A more detailed analysis of the correlation functions can provide clues about the nature of the non-Gaussianity.

Although correlation functions provide an excellent approach from a purely mathematical point of view, determining correlation functions for a given realization of a near-Gaussian field $h$ may not always be practical, as it requires precise measurements of $h$ in order to determine the correlation functions with a large enough precision.

In this paper, we consider two geometrical tests for Gaussianity, the first of which involves counting the number of maxima and minima. 


\section{MAXIMA VERSUS MINIMA}

Due to symmetry, a Gaussian field $H$ has as many minima as it has maxima. For a perturbed Gaussian field, like $h=H+$ $\varepsilon H^{2}$, this may no longer be the case. Therefore, the difference in densities of maxima and minima can serve as an indication of non-Gaussianity. We shall now derive what this difference is in the generic case of a field given by $h(\vec{r})=F_{N L}[H(\vec{r})]$, where $H$ is a Gaussian field and $F_{N L}$ is any (nonlinear) function (e.g., the identity plus a perturbation), which depends only on $H(\vec{r})$, i.e., the original (unperturbed) value of the field at that same point. This scheme we will refer to as a local perturbation.

Transforming the function with $F_{N L}$ does not move maxima and minima around, but it can interchange them, depending on the sign of $F_{N L}^{\prime}=d F_{N L} / d H$ at the point in question. To see this, note that maxima and minima, together with saddle points, are critical points. The critical points of $h$ are given by

$$
0=\vec{\nabla} h(\vec{r})=\frac{d h}{d H} \vec{\nabla} H(\vec{r})=F_{N L}^{\prime}(H) \vec{\nabla} H(\vec{r}) .
$$

We see that the critical points of $H$ and $h$ are the same points; however, the prefactor $F_{N L}^{\prime}(H)$ may influence the type of critical point. The three types can be distinguished by considering the second derivatives: saddle points have $h_{x x} h_{y y}-h_{x y}^{2}<0$, whereas for maxima and minima (together called extrema) this is positive. For maxima, unlike minima, we have $h_{x x}<0$ (or $\left.h_{y y}<0\right)$.

Consider a critical point $\overrightarrow{r_{0}}$ and let $z=H\left(\overrightarrow{r_{0}}\right)$. The second derivatives of $h$ at $\vec{r}_{0}$ simply have an extra factor $F_{N L}^{\prime}(z)$ as compared to the second derivatives of $H$. This has no influence on the sign of $h_{x x} h_{y y}-h_{x y}^{2}$, therefore, the saddle points (extrema) of $H$ are also saddle points (extrema) of $h$. However, a maximum (minimum) of $H$ is a minimum (maximum) of $h$ when $F_{N L}^{\prime}(z)<0$. In order to determine how many extrema will undergo such a transformation, we need to know how often $F_{N L}^{\prime}(z)<0$ at such points.

Let $g(z)$ be the probability density that a certain minimum $\overrightarrow{r_{0}}$ of $H$ has the value $H\left(\overrightarrow{r_{0}}\right)=z$. The probability $P$ that a minimum of $H$ becomes a maximum of $h$ is then

$$
P=\int_{z: F_{N L}^{\prime}(z)<0} d z g(z)
$$

For example, if we consider a square perturbation $h=H+$ $\varepsilon H^{2}$, for which $F_{N L}^{\prime}(z)=1+2 \varepsilon z$, we have

$$
P=\int_{-\infty}^{-\frac{1}{2 \varepsilon}} d z g(z)
$$

Because of the symmetry of $H$, the maxima are distributed according to $g(-z)$. With that, we can similarly define a probability $Q$ that a maximum becomes a minimum going from $H$ to $h$.

Let $n_{0}$ be the density of minima (or maxima) of $H$. The density of minima (maxima) of $H$ which are maxima (minima) of $h$ is then $P n_{0}\left(Q n_{0}\right)$. We can quantify the resulting imbalance in maxima and minima in the dimensionless parameter

$$
\begin{aligned}
\Delta n & \equiv \frac{n_{\max }-n_{\min }}{n_{\max }+n_{\min }}=\frac{(1+P-Q) n_{0}-(1-P+Q) n_{0}}{2 n_{0}} \\
& =P-Q=\int_{z: F_{N L}^{\prime}(z)<0} d z[g(z)-g(-z)] .
\end{aligned}
$$

Thus, if we can determine $g(z)$, we can calculate the exact imbalance between the maxima and minima of $h$.

\section{A. Distribution of minimum values}

\section{One dimension}

Let us first consider the probability distribution for minimum values of a Gaussian function on a line. We will then generalize to two dimensions, and afterward, discuss how these distributions depend on the power spectrum. We start with

$$
H(x)=\sum_{k} A(k) \cos \left(k x+\phi_{k}\right)
$$

The minima are given by $H_{x}\left(x_{0}\right)=0$ and $H_{x x}\left(x_{0}\right)>0$. We would thus like to know the probability density that $H\left(x_{0}\right)=z$, given that $H_{x}\left(x_{0}\right)=0$ and $H_{x x}\left(x_{0}\right)>0$ :

$$
\begin{aligned}
g(z) & =p\left[H\left(x_{\min }\right)=z\right] \\
& =\frac{1}{n} p\left[H\left(x_{0}\right)=z \wedge H_{x}\left(x_{0}\right)=0 \wedge H_{x x}\left(x_{0}\right)>0\right] .
\end{aligned}
$$

Here, $n \equiv p\left[H_{x}\left(x_{0}\right)=0 \wedge H_{x x}\left(x_{0}\right)>0\right]$ can be identified as the density of the minima. We need to determine the joint probability distribution $p\left[H\left(x_{0}\right), H_{x}\left(x_{0}\right), H_{x x}\left(x_{0}\right)\right]$; since $H$ is homogeneous, $p$ does not depend on $x_{0}$.

Let us take a closer look at the first derivative

$$
\begin{aligned}
H_{x}\left(x_{0}\right) & =\sum_{k} A(k)(-k) \sin \left(k x_{0}+\phi_{k}\right) \\
& =\sum_{k} k A(k) \cos \left(k x_{0}+\phi_{k}+\frac{1}{2} \pi\right) .
\end{aligned}
$$

We see that the expression for $H_{x}$ still describes a Gaussian: the phases are simply increased by $\frac{1}{2} \pi$ (modulo $2 \pi$ ) and the spectrum has picked up a factor of $k$. The bottom line is that $H_{x}\left(x_{0}\right)$ is a Gaussian variable, and it is easy to confirm that the same goes for $H_{x x}\left(x_{0}\right)$ (or any derivative).

We thus have three Gaussian variables. The joint probability distribution of a set of (correlated) Gaussian random variables is given by [compare Eq. (2)]

$$
p\left(\xi_{1}, \ldots, \xi_{n}\right)=\frac{1}{(2 \pi)^{n / 2} \sqrt{\operatorname{det} C}} \exp \left(-\frac{1}{2} \sum_{i, j}\left(C^{-1}\right)_{i j} \xi_{i} \xi_{j}\right) .
$$

Moreover, the coefficients $C$ can be determined measuring the statistics of the field: it is the matrix of correlations

$$
C_{i j}=\left\langle\xi_{i} \xi_{j}\right\rangle
$$

Let us calculate $\left\langle H(x) H_{x x}(x)\right\rangle$ as an example. Again, homogeneity allows us to set $x_{0}=0$ for convenience. 
We then find

$$
\begin{aligned}
\left\langle H\left(x_{0}\right) H_{x x}\left(x_{0}\right)\right\rangle & =\left\langle H(0) H_{x x}(0)\right\rangle \\
& =\left\langle\sum_{k} A(k) \cos \phi_{k} \sum_{k^{\prime}} A\left(k^{\prime}\right)\left(-k^{\prime 2}\right) \cos \phi_{k^{\prime}}\right\rangle \\
& =\sum_{k k^{\prime}} A(k) A\left(k^{\prime}\right)\left(-k^{\prime 2}\right)\left\langle\cos \phi_{k} \cos \phi_{k^{\prime}}\right\rangle \\
& =\sum_{k k^{\prime}} A(k) A\left(k^{\prime}\right)\left(-k^{\prime 2}\right) \frac{1}{2} \delta_{k k^{\prime}} \\
& =\sum_{k}-\frac{1}{2} A(k)^{2} k^{2}=-K_{2} .
\end{aligned}
$$

Here, we made use of the moment $K_{2}$ defined in Eq. (A7).

An even and an odd derivative of $H$ are always uncorrelated, e.g.,

$$
\begin{aligned}
\left\langle H(0) H_{x}(0)\right\rangle & =\sum_{k k^{\prime}} A(k) A\left(k^{\prime}\right)\left(-k^{\prime}\right)\left\langle\cos \phi_{k} \sin \phi_{k^{\prime}}\right\rangle \\
& =\sum_{k k^{\prime}} A(k)^{2}(-k)\left\langle\cos \phi_{k} \sin \phi_{k}\right\rangle \delta_{k k^{\prime}}=0 .
\end{aligned}
$$

This is because an even derivative features cosines while an odd derivative has sines, and their product averages to zero, as above.

The final result is that for $H, H_{x}$, and $H_{x x}$ the correlations are

$$
C=\left(\begin{array}{ccc}
1 & 0 & -K_{2} \\
0 & K_{2} & 0 \\
-K_{2} & 0 & K_{4}
\end{array}\right)
$$

The determinant of $C$ is $K_{2}\left(K_{4}-K_{2}^{2}\right)$ and its inverse is

$$
C^{-1}=\frac{1}{K_{2}\left(K_{4}-K_{2}^{2}\right)}\left(\begin{array}{ccc}
K_{2} K_{4} & 0 & K_{2}^{2} \\
0 & K_{4}-K_{2}^{2} & 0 \\
K_{2}^{2} & 0 & K_{2}
\end{array}\right) .
$$

This gives

$$
\begin{aligned}
p\left(H, H_{x}, H_{x x}\right)= & \frac{1}{(2 \pi)^{3 / 2} \sqrt{K_{2}\left(K_{4}-K_{2}^{2}\right)}} \\
& \times \exp \left(\frac{H_{x}^{2}}{2 K_{2}}-\frac{K_{4} H^{2}+2 K_{2} H H_{x x}+H_{x x}^{2}}{2\left(K_{4}-K_{2}^{2}\right)}\right) .
\end{aligned}
$$

The plan is now to set $H=z$ and $H_{x}=0$ and integrate $p$ over $H_{x x}$. However, one important factor still needs to be added. The probability we have calculated is actually a probability density [since the probability that $H^{\prime}\left(x_{0}\right)=0$ and $H\left(x_{0}\right)=z$ exactly is zero], and it is not defined with respect to the variables we need. It is defined by fixing a point $x_{0}$ and determining the probability that $H_{x}$ vanishes within a certain tolerance at that point:

$$
\frac{P\left[H\left(x_{0}\right) \in[z, z+d z] \wedge H_{x}\left(x_{0}\right) \in\left[0, d H^{\prime}\right]\right]}{d z d H^{\prime}} .
$$

Instead, we actually want the probability that there is an exact critical point within a certain distance of $x_{0}$ :

$$
\frac{P\left(\begin{array}{c}
\exists x_{m} \in\left[x_{0}, x_{0}+d x\right]: \\
H\left(x_{m}\right) \in[z, z+d z] \wedge H_{x}\left(x_{m}\right)=0
\end{array}\right)}{d x d z} .
$$

Over the range $d x, d H^{\prime}$ varies by

$$
d H^{\prime}=\left|\frac{\partial H_{x}}{\partial x}\right| d x=\left|H_{x x}\right| d x .
$$

In order to get the desired probability density with respect to $x$, we need to multiply our current probability density with $\left|H_{x x}\right|$.

The probability distribution for the minima is thus given by [see Eq. (10)]

$$
g(z)=\frac{1}{n} \int_{0}^{\infty} d H_{x x} p\left(H=z, H_{x}=0, H_{x x}\right)\left|H_{x x}\right| .
$$

The prefactor, featuring the density of minima $n$, can be regarded as a normalization constant and is found by integrating $g(z)$ over the entire $z$ range. This is easily accomplished by taking the expression above and first integrate over $z$, and only then over $H_{x x}$. The result is

$$
\int_{-\infty}^{\infty} d z g(z)=1 \Rightarrow n=\frac{1}{2 \pi} \sqrt{K_{4} / K_{2}} .
$$

The integrand in Eq. (20) is also Gaussian, but it is only integrated over for positive $H_{x x}$, resulting in

$$
\begin{aligned}
g(z)= & \sqrt{\frac{1-\lambda}{2 \pi}} \exp \left(-\frac{1}{2(1-\lambda)} z^{2}\right) \\
& -\frac{1}{2} \sqrt{\lambda} z \exp \left(-\frac{1}{2} z^{2}\right) \operatorname{erfc}\left(\sqrt{\frac{\lambda}{2(1-\lambda)} z}\right) .
\end{aligned}
$$

Here, erfc is the complementary error function

$$
\operatorname{erfc}(x) \equiv \frac{2}{\sqrt{\pi}} \int_{x}^{\infty} d t e^{-t^{2}},
$$

which converges to 1 as $x$ goes to $-\infty$. The two parameters $K_{2}$ and $K_{4}$ have been merged into a single dimensionless parameter

$$
\lambda \equiv \frac{K_{2}^{2}}{K_{4}} \quad(0 \leqslant \lambda \leqslant 1) .
$$

Note that we set $K_{0} \equiv\left\langle H^{2}\right\rangle=1$ for convenience. In the generic case $K_{0} \neq 1$, we have $\lambda=K_{2}^{2} /\left(K_{0} K_{4}\right)$. A proof that $\lambda \leqslant 1$ is derived explicitly in the next section.

\section{Two dimensions}

In two dimensions, the procedure to calculate the distribution of the minima is similar. The minima are defined by the conditions $H_{x}=H_{y}=0$ (defining critical points), $H_{x x} H_{y y}-H_{x y}^{2}>0$ (separating extrema from saddle points), and $H_{x x}, H_{y y}>0$ (distinguishing minima from maxima). We thus need to find $p\left(H, H_{x}, H_{y}, H_{x x}, H_{y y}, H_{x y}\right)$. This is still a Gaussian joint distribution function. 
We start again by determining the correlations, for example (again setting $\vec{r}=0$ for convenience),

$$
\begin{aligned}
\left\langle H_{x x} H_{y y}\right\rangle & =\sum_{\vec{k} \vec{k}^{\prime}} A(k) A\left(k^{\prime}\right) k_{x}^{2} k_{y}^{\prime 2}\left\langle\cos \phi_{\vec{k}} \cos \phi_{\vec{k}^{\prime}}\right\rangle \\
& =\sum_{\vec{k} \vec{k}^{\prime}} A(k) A\left(k^{\prime}\right) k_{x}^{2} k_{y}^{\prime 2} \frac{1}{2} \delta_{\vec{k} \vec{k}^{\prime}}=\sum_{\vec{k}} \frac{1}{2} A(k)^{2} k_{x}^{2} k_{y}^{2} \\
& =\frac{1}{2 \pi} \int_{0}^{2 \pi} \int_{0}^{\infty} d k d \theta \Pi(k) k^{4} \cos ^{2} \theta \sin ^{2} \theta \\
& =\frac{1}{8} \int_{0}^{\infty} d k \Pi(k) k^{4}=\frac{1}{8} K_{4} .
\end{aligned}
$$

In the third line we replaced the sum by an integral and performed it using polar coordinates.

Remember from the one-dimensional case that the correlation of an even and an odd derivative is always zero because in the calculation we encounter a product of a cosine and a sine, which integrated over the (random) phase yields zero. Based on the calculation method demonstrated above, we can make a more general statement: When the combined number of $x$ derivatives ( $y$ derivatives) is odd, the integral over $\theta$ (as above) features a cosine (sine) with an odd exponent; the integral over $\theta$ then gives zero. If we apply this rule to our six variables, we see that $H_{x}, H_{y}$, and $H_{x y}$ all have no "compatible match" in this respect; therefore, they are uncorrelated to all other variables. This allows us to factorize the joint probability distribution

$$
\begin{aligned}
& p\left(H, H_{x}, H_{y}, H_{x x}, H_{y y}, H_{x y}\right) \\
& \quad=p\left(H_{x}\right) p\left(H_{y}\right) p\left(H_{x y}\right) p\left(H, H_{x x}, H_{y y}\right) .
\end{aligned}
$$

The probability densities of the individual variables are straightforward,

$$
\begin{gathered}
p\left(H_{x}\right)=\frac{1}{\sqrt{\pi K_{2}}} \exp \left(-\frac{1}{K_{2}} H_{x}^{2}\right), \\
p\left(H_{y}\right)=\frac{1}{\sqrt{\pi K_{2}}} \exp \left(-\frac{1}{K_{2}} H_{y}^{2}\right), \\
p\left(H_{x y}\right)=\frac{2}{\sqrt{\pi K_{4}}} \exp \left(-\frac{4}{K_{4}} H_{x y}^{2}\right) .
\end{gathered}
$$

For $H, H_{x x}$, and $H_{y y}$, we determine the correlation matrix

$$
C=\left(\begin{array}{ccc}
1 & -\frac{1}{2} K_{2} & -\frac{1}{2} K_{2} \\
-\frac{1}{2} K_{2} & \frac{3}{8} K_{4} & \frac{1}{8} K_{4} \\
-\frac{1}{2} K_{2} & \frac{1}{8} K_{4} & \frac{3}{8} K_{4}
\end{array}\right) .
$$

The determinant of $C$ is $\frac{1}{8} K_{4}\left(K_{4}-K_{2}^{2}\right)$ and its inverse is

$$
\begin{aligned}
C^{-1}= & \frac{1}{K_{4}\left(K_{4}-K_{2}^{2}\right)} \\
& \times\left(\begin{array}{ccc}
K_{4}^{2} & K_{2} K_{4} & K_{2} K_{4} \\
K_{2} K_{4} & 3 K_{4}-2 K_{2}^{2} & 2 K_{2}^{2}-K_{4} \\
K_{2} K_{4} & 2 K_{2}^{2}-K_{4} & 3 K_{4}-2 K_{2}^{2}
\end{array}\right) .
\end{aligned}
$$

After some rearranging, Eq. (12) gives

$$
\begin{aligned}
p\left(H, H_{x x}, H_{y y}\right)= & \frac{1}{\pi^{3 / 2} \sqrt{K_{4}\left(K_{4}-K_{2}^{2}\right)}} \\
& \times \exp \left(-\frac{\left(K_{4} H+K_{2} H_{x x}+K_{2} H_{y y}\right)^{2}}{2 K_{4}\left(K_{4}-K_{2}^{2}\right)}\right. \\
& \left.-\frac{\left(H_{x x}-H_{y y}\right)^{2}}{2 K_{4}}-\frac{H_{x x}^{2}+H_{y y}^{2}}{K_{4}}\right)
\end{aligned}
$$

As in the one-dimensional case, we now have a probability density with respect to $H_{x}$ and $H_{y}$, which we need to convert to one with respect to $x$ and $y$. For that we need to multiply $p$ with the Jacobian determinant

$$
\left|\frac{\partial\left(H_{x}, H_{y}\right)}{\partial(x, y)}\right|=\left|H_{x x} H_{y y}-H_{x y}^{2}\right| .
$$

The probability distribution for the minima is thus given by

$$
\begin{aligned}
g(z)= & \frac{1}{n} p\left(H_{x}=0\right) p\left(H_{y}=0\right) \\
& \times \iiint d H_{x x} d H_{y y} d H_{x y} p\left(H=z, H_{x x}, H_{y y}\right) \\
& \times p\left(H_{x y}\right)\left|H_{x x} H_{y y}-H_{x y}^{2}\right| \\
= & \frac{1}{n \pi K_{2}} \iiint d H_{x x} d H_{y y} d H_{x y} p\left(z, H_{x x}, H_{y y}\right) \\
& \times p\left(H_{x y}\right)\left|H_{x x} H_{y y}-H_{x y}^{2}\right| .
\end{aligned}
$$

The integrals must be taken over the volume for which $H_{x x} H_{y y}-H_{x y}^{2}>0$ and $H_{x x}, H_{y y}>0$, which forms the domain of the minima. These constraints and the integration can be simplified by making the following change of variables:

$$
\begin{aligned}
r \cos \theta & =\frac{1}{2}\left(H_{x x}-H_{y y}\right), \\
r \sin \theta & =H_{x y}, \\
s & =\frac{1}{2}\left(H_{x x}+H_{y y}\right), \\
d H_{x x} d H_{y y} d H_{x y} & =2 r d r d s d \theta .
\end{aligned}
$$

In terms of these new variables, we have $H_{x x} H_{y y}-H_{x y}^{2}=$ $s^{2}-r^{2}$ and the constraints of the volume are given by $0<$ $r<s$. We get

$$
\begin{aligned}
g(z)= & \frac{1}{n \pi K_{2}} \int_{0}^{2 \pi} \int_{0}^{\infty} \int_{0}^{s} d r d s d \theta \frac{4 r\left(s^{2}-r^{2}\right)}{\pi^{2} K_{4} \sqrt{K_{4}-K_{2}^{2}}} \\
& \times \exp \left(-\frac{K_{4} z^{2}+4 K_{2} s z+4 s^{2}}{2\left(K_{4}-K_{2}^{2}\right)}-\frac{4 r^{2}}{K_{4}}\right) .
\end{aligned}
$$

The density of the minima $n$ can again readily be obtained by integrating over $z$ :

$$
\int_{-\infty}^{\infty} d z g(z)=1 \Rightarrow n=\frac{K_{4}}{8 \sqrt{3} \pi K_{2}} .
$$

Note that this result matches the one obtained in [6]. 
After evaluating the double integral (taking care to integrate over $r$ first), we obtain

$$
\begin{aligned}
& g(z)=\sqrt{\frac{3}{2 \pi(3-2 \lambda)}} \exp \left(-\frac{3}{2(3-2 \lambda)} z^{2}\right) \\
& \times \operatorname{erfc}\left(\sqrt{\frac{\lambda}{2(1-\lambda)(3-2 \lambda)}} z\right)
\end{aligned}
$$

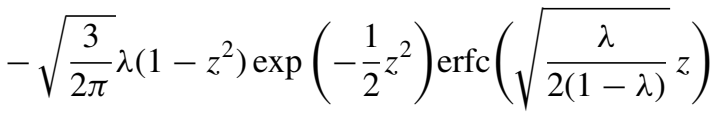

$$
\begin{aligned}
& -\frac{1}{\pi} \sqrt{3 \lambda(1-\lambda)} z \exp \left(-\frac{1}{2(1-\lambda)} z^{2}\right) \text {. }
\end{aligned}
$$

The two parameters $K_{2}$ and $K_{4}$ have been merged into one as before:

$$
\lambda \equiv \frac{K_{2}^{2}}{K_{4}} \quad(0 \leqslant \lambda \leqslant 1) .
$$

Again, when we set $K_{0}=\left\langle H^{2}\right\rangle \neq 1$, we get $\lambda=K_{2}^{2} /\left(K_{0} K_{4}\right)$.

Let us prove that $\lambda \leqslant 1$. After some rearranging, we see that this is equivalent to $K_{0} K_{4}-K_{2}^{2} \geqslant 0$. We find

$$
K_{0} K_{4}-K_{2}^{2}=\iint d k d k^{\prime} \Pi(k) \Pi\left(k^{\prime}\right)\left(k^{\prime 4}-k^{2} k^{\prime 2}\right) .
$$

Note that we could just as well replace $k^{\prime 4}$ with $k^{4}$ (because everything else is symmetric in $k$ and $k^{\prime}$ ), and hence also with $\frac{1}{2}\left(k^{4}+k^{\prime 4}\right)$. If we do the latter, we can rewrite

$$
\frac{1}{2}\left(k^{4}+k^{\prime 4}\right)-k^{2} k^{\prime 2}=\frac{1}{2}\left(k-k^{\prime}\right)^{2} .
$$

We see that this is positive, together with $\Pi(k)$ and $\Pi\left(k^{\prime}\right)$, hence the integrand is positive and the integral too, which concludes the proof.

We have compared Eq. (37) with distributions obtained from computer-generated Gaussian fields; details about these numerical simulations and how the minima were identified can be found in Appendix B. As can be seen in Fig. 2, the agreement between Eq. (37) and the numeric results is excellent.

Let us take a closer look at Eq. (37). The two limits of $\lambda$ give results with interesting physical interpretations:

$$
\begin{aligned}
& \lim _{\lambda \rightarrow 0} g(z)=\frac{1}{\sqrt{2 \pi}} e^{-\frac{1}{2} z^{2}}-\sqrt{\lambda} \frac{4}{\sqrt{3} \pi} z e^{-\frac{1}{2} z^{2}}+O(\lambda), \\
& \lim _{\lambda \rightarrow 1} g(z)=(1-\operatorname{sgn} z) \sqrt{\frac{3}{2 \pi}}\left(e^{-z^{2}}-1+z^{2}\right) e^{-\frac{1}{2} z^{2}} .
\end{aligned}
$$

The case $\lambda=0$ occurs when $K_{4}$ is unbounded [e.g., when $\Pi(k)$ scales as $k^{-6}$ ]. We see that the distribution is then an elementary Gaussian. A rough intuitive explanation for this is as follows. The key feature of this limit is that the maxima and minima arise from very rapid oscillations that are superimposed on top of a slowly varying field. In fact, if $K_{4}$ is extremely large, the waves with a short wavelength (large $|\vec{k}|$ ) have an amplitude that is small, but not negligible. They therefore create large fluctuations in the gradient of the field and hence a lot of extrema, a fact that can also be seen from Eq. (36). Meanwhile, the height of the surface at any point (including the abundant minima) is dominated by the waves with a large amplitude, which have long wavelengths (small $|\vec{k}|$ ). The location of the minima and the height of the surface are thus independent.

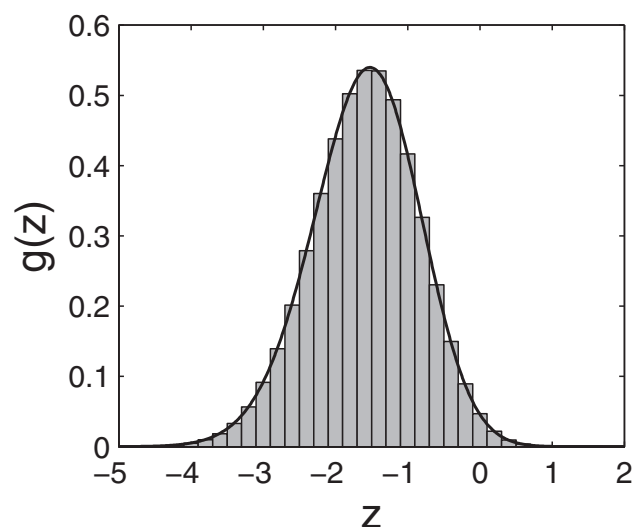

(a)

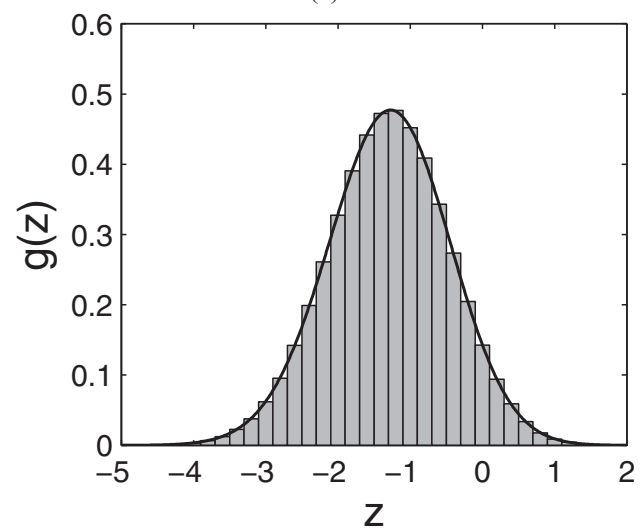

(b)

FIG. 2. Histograms of the values of $10^{6}$ minima obtained from simulations, together with the distribution given by Eq. (37), for (a) a disk spectrum $\left(\lambda=\frac{3}{4}\right)$; (b) a Gaussian spectrum $\left(\lambda=\frac{1}{2}\right)$.

Therefore, the distribution of the value of $H$ at a minimum is the same as for any other point: Gaussian.

Now we consider $\lambda=1$. From our proof that $\lambda \leqslant 1$, it is not hard to see that this can only occur when $\Pi(k)=\delta\left(k-k_{0}\right)$ for some constant $k_{0}$. This is called a ring spectrum since the only occurring wave vectors are the ones with $|\vec{k}|=k_{0}$, which describes a circle in $\vec{k}$ space. Inspecting Eq. (42) we see that, due to the factor $(1-\operatorname{sgn} z)$, all minima have a negative value of $H$, as the simulations also show (see Fig. 3). The explanation is that height fields with a ring spectrum necessarily satisfy $\nabla^{2} H=-k_{0}^{2} H$, therefore, if $H$ is positive, the mean curvature $H_{x x}+H_{y y}<0$, so the point can not be a minimum. In other words, such Gaussian fields are random solutions to Helmholtz's equation: they could represent the height field of a large membrane resonating at a certain frequency but with some randomness preventing a particular mode among the many at that frequency from stabilizing.

While Eq. (37) appears quite complex, some of its parameters have more transparent forms. The expectation value $\mu$ and standard deviation $\sigma$, for example, are

$$
\begin{gathered}
\mu=-4 \sqrt{\frac{2}{3 \pi} \lambda}, \\
\sigma=\sqrt{1-\frac{32-(6 \sqrt{3}-2) \pi}{3 \pi} \lambda .} .
\end{gathered}
$$




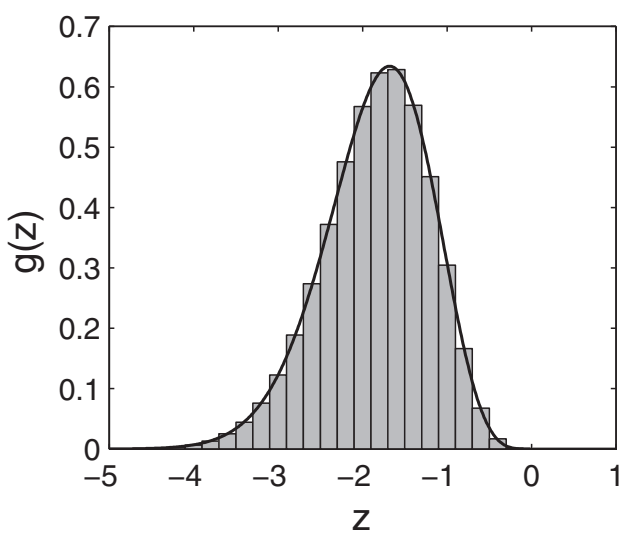

FIG. 3. Histogram of the values of $10^{6}$ minima obtained from simulations, together with the distribution given by Eq. (37), for a ring spectrum $(\lambda=1)$. No minima with a positive value of $H$ were found.

When looking at Fig. 2, it appears that the distribution is itself almost Gaussian. This can be captured in the skewness $\gamma_{1}$ and kurtosis $\gamma_{2}$ :

$$
\begin{aligned}
\gamma_{1} & \equiv \frac{\mu_{3}}{\sigma^{3}}=-\frac{4 \sqrt{2}[64-(18 \sqrt{3}-11) \pi]}{\left\{3 \pi \lambda^{-1}-[32-(6 \sqrt{3}-2) \pi]\right\}^{3 / 2}} \\
& =-\frac{3.46}{\left(9.42 \lambda^{-1}-5.63\right)^{3 / 2}}, \\
\gamma_{2} \equiv & \frac{\mu_{4}}{\sigma^{4}}-3 \\
& =\frac{4\left[-1536+32(18 \sqrt{3}-11) \pi+9(2 \sqrt{3}-9) \pi^{2}\right]}{\left\{3 \pi \lambda^{-1}-[32-(6 \sqrt{3}-2) \pi]\right\}^{2}} \\
& =\frac{2.68}{\left(9.42 \lambda^{-1}-5.63\right)^{2}} .
\end{aligned}
$$

Here, $\mu_{n}$ is the $n$th moment about the mean: $\mu_{n} \equiv\langle(\xi-$ $\left.\langle\xi\rangle)^{n}\right\rangle$. The skewness is a measure of the symmetry of a distribution around the mean, while the kurtosis gives an indication of its "peakiness." For a Gaussian distribution, both the skewness and the kurtosis are zero. They can therefore be considered as a measure of the Gaussianity of a distribution; note, however, that a distribution is not necessarily Gaussian if both parameters are zero. The two parameters are shown in Fig. 4. Naturally, they both go to zero for $\lambda \rightarrow 0$.

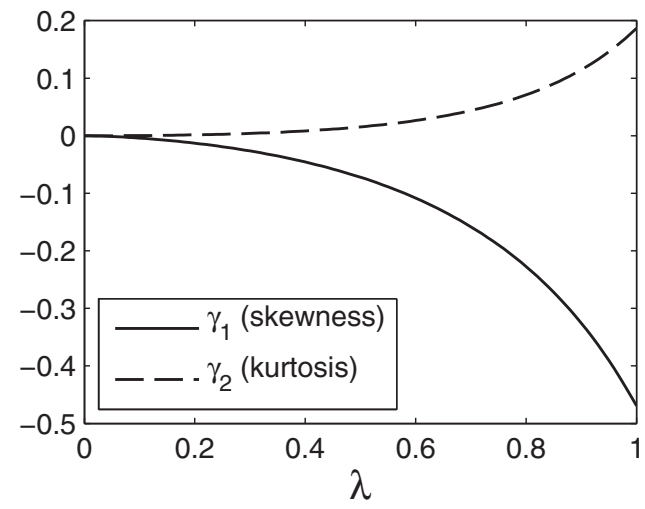

FIG. 4. The skewness $\left(\gamma_{1}\right)$ and kurtosis $\left(\gamma_{2}\right)$ of the distribution [Eq. (37)] as a function of $\lambda$ [see Eqs. (45) and (46)].

\section{B. Maxima and minima imbalance}

Now that we have obtained $g(z)$, we can calculate the relative imbalance between the densities of maxima and minima of $h=F_{N L}(H)$, in accordance with Eq. (8):

$$
\Delta n \equiv \frac{n_{\max }-n_{\min }}{n_{\max }+n_{\min }}=\int_{z: F_{N L}^{\prime}(z)<0} d z[g(z)-g(-z)] .
$$

The most basic example of a perturbed Gaussian for which we may expect $\Delta n \neq 0$ is $h=H+\varepsilon H^{2}$. In this case, the domain of integration is $\left[-\infty,-\frac{1}{2 \varepsilon}\right]$. We have compared Eq. (47) with results from computer-generated fields, for two different spectra; in Fig. 5 a so-called disk spectrum was used:

$$
A(k)^{2} \sim \theta\left(k_{0}-k\right), \quad K_{2 n}=\frac{k_{0}^{2 n}}{n+1}, \quad \lambda=\frac{3}{4} .
$$

Figure 6 features results for a Gaussian spectrum:

$$
A(k)^{2} \sim \exp \left(-k^{2} / 2 k_{0}^{2}\right), \quad K_{2 n}=2^{n} n ! k_{0}^{2 n}, \quad \lambda=\frac{1}{2} .
$$

In both cases, we see an excellent agreement between the results from the simulations and our theoretical formula.

In both figures, we see that $\Delta n$ increases dramatically starting $\varepsilon \sim 0.15$. This can be explained intuitively as follows: the balance in densities of maxima and minima is disturbed by extrema located below $H=-\frac{1}{2 \varepsilon}$. Since $H$ is a standard Gaussian, such low values (i.e., large negative values) of $H$ are exponentially rare. It is only when $-\frac{1}{2 \varepsilon}$ is in the order of -1 that a significant $\Delta n$ can be expected. To get a rough estimate for the number of these extrema, we can just look at the density

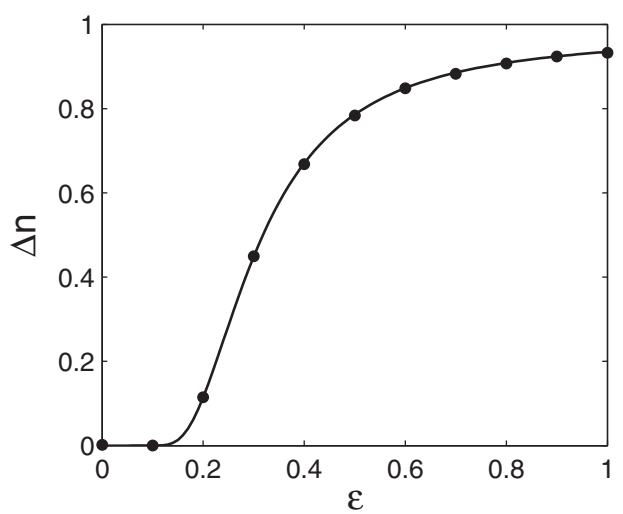

(a)

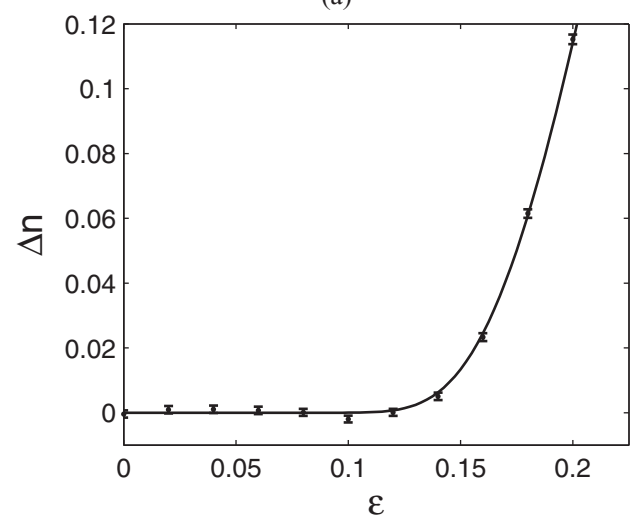

(b)

FIG. 5. $\Delta n$ for $h=H+\varepsilon H^{2}$ as a function of $\varepsilon$, where $H$ has a disk spectrum $\left(\lambda=\frac{3}{4}\right)$. The data points stem from simulations, the solid curve is Eq. (47). The two graphs are for different ranges of $\varepsilon$. 


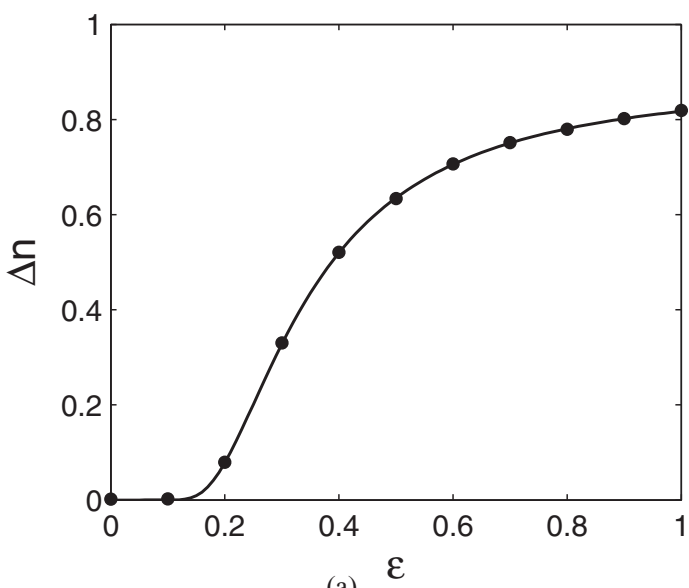

(a)

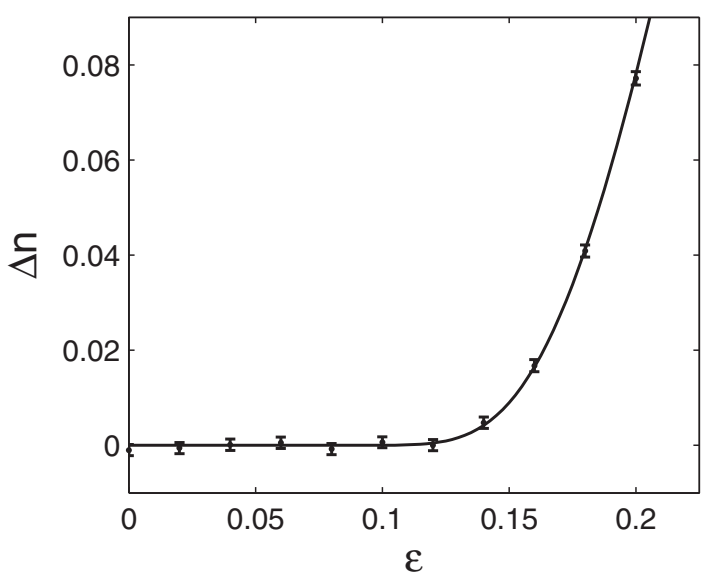

(b)

FIG. 6. $\Delta n$ for $h=H+\varepsilon H^{2}$ as a function of $\varepsilon$, where $H$ has a Gaussian spectrum $\left(\lambda=\frac{1}{2}\right)$. The data points stem from simulations, the solid curve is Eq. (47). The two graphs are for different ranges of $\varepsilon$.

of points with $H=-\frac{1}{2 \varepsilon}$ (ignoring the requirement that they be minima does not change the exponential dependence). This is $e^{-1 /\left(8 \varepsilon^{2}\right)}$. A more careful approximation (see Appendix C) gives $\Delta n \sim \sqrt{\frac{3}{2 \pi}} \frac{\lambda}{\varepsilon} e^{-\frac{1}{8 \varepsilon^{2}}}$.

This argument also applies to the generic case $h=H+$ $\varepsilon f_{N L}(H)$, where $f_{N L}$ designates a perturbation and $\varepsilon$ is a parameter controlling the size of the perturbation. Now, $\varepsilon f_{N L}^{\prime}(H)$ needs to be in the order of 1 for $\Delta n$ to be significantly nonzero. Thus, measuring the imbalance between maxima and minima does not give a very sensitive test of the type of non-Gaussianity that we have considered here, in the limit of small $\varepsilon$. However, Eq. (47) is a nonperturbative result that also holds for large $\varepsilon$.

\section{UMBILICAL POINTS}

Umbilical points are points on a surface where the curvature of the surface is the same along all directions. The curvature depicts how much the surface bends along a given direction, just like the second derivative of a one-dimensional function does. At an umbilical point then, the surface is locally spherical (or flat).
In order to make a proper mathematical formulation, consider a two-dimensional function $f(x, y)$. We consider any specific point $\left(x_{0}, y_{0}\right)$ and any direction given by an angle $\psi$. Along this direction, the function can be parametrized as

$$
f_{\psi}(r)=f\left(x_{0}+r \cos \psi, y_{0}+r \sin \psi\right) .
$$

This function now describes what $f$ looks like at $\left(x_{0}, y_{0}\right)$ along the direction $\psi$. The curvature is the value of the second derivative of $f_{\psi}(r)$ at $r=0$,

$$
\begin{aligned}
f_{\psi}^{\prime \prime}(0)= & \left.\frac{d^{2} f_{\psi}}{d r^{2}}\right|_{r=0}=f_{x x}\left(x_{c}, y_{c}\right) \cos ^{2} \psi+f_{y y}\left(x_{c}, y_{c}\right) \sin ^{2} \psi \\
& +2 f_{x y}\left(x_{c}, y_{c}\right) \sin \psi \cos \psi \\
= & \left(\frac{1}{2}+\frac{1}{2} \cos 2 \psi\right) f_{x x}+\left(\frac{1}{2}-\frac{1}{2} \cos 2 \psi\right) f_{y y} \\
& +\sin 2 \psi f_{x y} \\
= & \frac{1}{2}\left(f_{x x}+f_{y y}\right)+\frac{1}{2}\left(f_{x x}-f_{y y}\right) \cos 2 \psi+f_{x y} \sin 2 \psi
\end{aligned}
$$

We can write this in a more lucid form by applying the transformation

$$
\begin{aligned}
\frac{1}{2}\left(f_{x x}-f_{y y}\right) & =R \cos \alpha, \quad R=\frac{1}{2} \sqrt{\left(f_{x x}-f_{y y}\right)^{2}+4 f_{x y}^{2}}, \\
f_{x y} & =R \sin \alpha, \quad \tan \alpha=\frac{2 f_{x y}}{f_{x x}-f_{y y}} .
\end{aligned}
$$

With this, we find

$$
\begin{aligned}
f_{\psi}^{\prime \prime}(0)= & \frac{1}{2}\left(f_{x x}+f_{y y}\right) \\
& +\frac{1}{2} \sqrt{\left(f_{x x}-f_{y y}\right)^{2}+4 f_{x y}^{2}} \cos (2 \psi-\alpha) .
\end{aligned}
$$

With the curvature now properly defined, we introduce the two principal directions, which are the directions along which the curvature is maximal or minimal. The corresponding curvatures are known as the principal curvatures. We can easily see from Eq. (53) that these two directions are given by $2 \psi-\alpha=k \pi$ and hence perpendicular to each other.

As noted before, at an umbilical point the curvature is the same along all directions. In other words, the two principal curvatures are the same, and the principal directions can not be defined. From Eq. (53), the definition of an umbilical point is easily seen to be

$$
f_{x x}=f_{y y} \quad \text { and } \quad f_{x y}=0 .
$$

Umbilical points can be classified in three types. The distinction can be clearly made when one looks at the curvature lines. These are curves which are always tangent to a principal direction, either the one corresponding with the maximal curvature or the minimum one. These two sets of curvature lines intersect at right angles since as noted before, the principal directions are always perpendicular to each other. At an umbilical point, no principal direction can be defined, giving one of the three patterns shown in Fig. 7. There are three types: lemons, monstars, and stars.

We see that, in each case, the umbilical point is a topological defect, having a topological index (see [30], for example). 


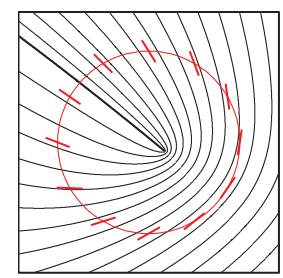

(a)

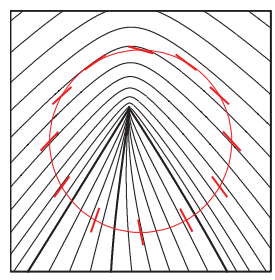

(b)

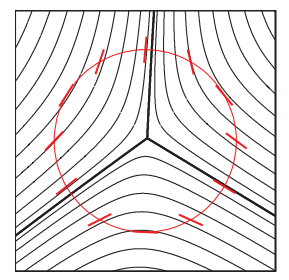

(c)
FIG. 7. (Color online) One set of curvature lines (black) around (a) a lemon, (b) a monstar, and (c) a star. The other set shows the same pattern in all cases. The red circle and line segments show how the principal direction rotates around the umbilical point.

Formally, the topological index is defined as

$$
n=\frac{1}{2 \pi} \oint \nabla \psi \cdot d l,
$$

where the path integral is taken over an (infinitesimal) counterclockwise loop around the defect. In words, it counts the number of revolutions the principal direction makes when traversing this closed loop. We see from Fig. 7 that, for the point labeled star, the direction makes half a clockwise rotation, which means a topological index of $-\frac{1}{2}$. The minus sign reflects that it rotates in the opposite direction with respect to the direction the loop is traversed in. The other two umbilical points have index $+\frac{1}{2}$.

Another characteristic separating the three is the number of curvature lines that terminate at the umbilical point. For a lemon, this is one, whereas for the other two it is three. We see that the third type of umbilical point shares properties with both others: it has topological index $+\frac{1}{2}$, as does a lemon, and three curvature lines terminating at it, like a star. This in-between nature of the point is reflected in its name: monstar.

The three types of umbilical point can also be distinguished using the third derivatives, much like the various types of critical points can be identified by the second derivatives. From Eqs. (52) and (53), we see that the two principal directions, for which the curvature is maximal or minimal, are given by

$$
\tan 2 \psi=\frac{2 f_{x y}}{f_{x x}-f_{y y}} .
$$

Note that the directions are given by angles modulo $\pi$, so this equation has two solutions: the two principal directions. The angle $2 \psi$ can be pictured as the argument of the vector $\vec{v}=\left({ }^{f_{x x}} \bar{f}_{x y} f_{y y}\right)$. At an umbilical point, both vector components are zero (hence, the principal directions are not defined). In order to determine the topological index, we need to know what the principal directions are in close proximity to this point, in order to evaluate the infinitesimal loop in Eq. (55). We can expand $\vec{v}$ using the third derivatives. For a point $\vec{r}$ near an umbilical point $\overrightarrow{r_{0}}$ we have

$$
\vec{v}=\left(\begin{array}{cc}
f_{x x x}-f_{y y x} & f_{x x y}-f_{y y y} \\
2 f_{x y x} & 2 f_{x y y}
\end{array}\right)\left(\begin{array}{c}
x-x_{u} \\
y-y_{u}
\end{array}\right)=\mathbf{A}\left(\vec{r}-\vec{r}_{0}\right) .
$$

If $\mathbf{A}$ were the identity matrix, then a counterclockwise loop around $\overrightarrow{r_{0}}$ would obviously result in $2 \psi$ increasing by $2 \pi$, giving index $+\frac{1}{2}$. In general, A may shear and rotate $\vec{r}$, or may reflect it. The former would have no effect on the charge. However, if $\mathbf{A}$ includes a reflection, the gradient would rotate in the opposite direction and the index becomes $-\frac{1}{2}$. Whether A describes a reflection or not is encoded in the sign of its determinant,

$$
\frac{1}{2} \operatorname{det} \mathbf{A}=\left(f_{x x x}-f_{x y y}\right) f_{x y y}+\left(f_{y y y}-f_{x x y}\right) f_{x x y} .
$$

Hence, the index of the umbilical point is $+\frac{1}{2}\left(-\frac{1}{2}\right)$ if $\operatorname{det} \mathbf{A}$ is positive (negative). Introducing $\alpha=f_{x x x}, \beta=f_{x x y}, \gamma=$ $f_{x y y}, \delta=f_{y y y}$, we thus find (see also [7])

$$
\alpha \gamma-\gamma^{2}+\beta \delta-\beta^{2} \begin{cases}>0 & \text { for } \mathrm{L}, \mathrm{M}, \\ <0 & \text { for } \mathrm{S} .\end{cases}
$$

As mentioned before, the criterion separating the lemons from the monstars (and stars), is the number of (locally straight) lines ending at the umbilical point: one for lemons, three for (mon)stars [that is one (three) for each principal direction]. This can also be expressed in terms of $\alpha, \beta, \gamma$, and $\delta$. Consider again a point $\vec{r}$ near $\overrightarrow{r_{0}}$. The principal directions are given by $\psi$ modulo $\frac{1}{2} \pi$, hence one of the two is directed toward the umbilical point when the argument $\theta$ of $\vec{r}-\overrightarrow{r_{0}}$ is equal to $\psi$ at $\vec{r}$, modulo $\frac{1}{2} \pi$.

To find an algebraic statement of this condition, we double both sides: $2 \theta \equiv 2 \psi(\bmod \pi)$. The right-hand side is the argument of $\vec{v}$ by Eq. (56). We can also find a vector whose argument is given by the left-hand side: let $\left(\begin{array}{l}x \\ y\end{array}\right)=\vec{r}-\overrightarrow{r_{0}}$; then $2 \theta$ is the argument of the vector $\left(x^{2}-y^{2}\right)$ : this is easily seen by mapping $\left(\begin{array}{l}x \\ y\end{array}\right)$ to the complex number $x+i y$ and taking its square, which doubles the argument. The condition for $\vec{r}$ being on a terminating curvature line is that the arguments of these two vectors must match modulo $\pi$, which translates to $\left(\begin{array}{c}x^{2}-y^{2} \\ 2 x y\end{array}\right)$ and $\mathbf{A}\left(\begin{array}{l}x \\ y\end{array}\right)$ being parallel to each other. This condition can be mathematically expressed using the matrix $\mathbf{A}$ from before and the cross product, giving

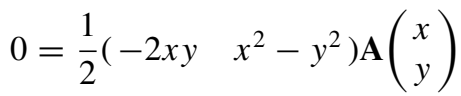

$$
\begin{aligned}
& =\frac{1}{2}\left(\begin{array}{ll}
-2 x y & x^{2}-y^{2}
\end{array}\right)\left(\begin{array}{c}
(\alpha-\gamma) x-(\delta-\beta) y \\
2 \beta x+2 \gamma y
\end{array}\right) \\
& =\beta x^{3}-(\alpha-2 \gamma) x^{2} y+(\delta-2 \beta) x y^{2}-\gamma y^{3} \text {. }
\end{aligned}
$$

Note that this equation describes lines passing through $\vec{r}_{0}$, whereas the curvature lines actually terminate on the defect. On one side of $\overrightarrow{r_{0}}$, this line corresponds with the line of maximal curvature, on the other side with minimal curvature. This is easily seen from Eq. (51), if one notes that the second derivatives change sign when passing through $\vec{r}_{0}$.

The number of straight lines passing through $\vec{r}_{0}$ is thus equal to the number of (real) roots of this cubic equation (that is, by interpreting this as an equation in $x / y$ ). This is captured by the discriminant: if it is positive, then there are three roots; if 
it is negative, there is only one. This results in (see also [7])

$$
\begin{aligned}
& 4\left[3 \gamma(\alpha-2 \gamma)-(\delta-2 \beta)^{2}\right] \\
& \times\left[3 \beta(\delta-2 \beta)-(\alpha-2 \gamma)^{2}\right] \\
& -[(\delta-2 \beta)(\alpha-2 \gamma)-9 \beta \gamma]^{2}
\end{aligned}\left\{\begin{array}{l}
>0 \text { for } \mathrm{M}, \mathrm{S}, \\
<0 \text { for L. }
\end{array}\right.
$$

According to the Poincaré-Hopf theorem, for any surface the total sum of all topological indices equals the Euler characteristic of the underlying manifold. For the two-dimensional plane that we consider, this is simply zero. As a consequence, the density of stars (with index $+\frac{1}{2}$ ) equals the combined density of lemons and monstars (with index $-\frac{1}{2}$ ). In other words, the star fraction, that is the density of stars divided by the total density of umbilical points, is always $\frac{1}{2}$. There is, however, no topological constraint on the lemon and monstar fractions. For isotropic Gaussian random fields, however, it has been shown that the monstar fraction is $\alpha_{M}=\frac{1}{2}-1 / \sqrt{5}=$ $0.053[7,16]$, independent of the spectrum of the Gaussian field. As a consequence, the monstar fraction promises to be a good criterion to test the (non-)Gaussianity of a field. Should one be given a random field, and find that the monstar fraction is not equal to 0.053 , one can immediately conclude that the field is not an isotropic Gaussian one.

\section{A. Monstar fraction}

We take a non-Gaussian field $h$ that can be described as a Gaussian field $H$ with a perturbation $f(H)$ added to it, and calculate how much the monstar fraction $\alpha_{M}$ deviates from the universal value 0.053 as a function of the perturbation $f(H)$. Our result also allows us to attack the reverse problem: when given a non-Gaussian field of which the type of perturbation is known, we can determine the monstar fraction and thus reveal the size of the perturbation.

As we have seen in Eqs. (54), (59), and (61), the monstars can be defined using the second and third derivatives of the field $h$ with respect to $x$ and $y$. Determining the monstar fraction thus boils down to determining how likely it is that at a specific point $\vec{r}$ the third derivatives $\alpha=h_{x x x}(\vec{r}), \beta=h_{x x y}(\vec{r})$, $\gamma=h_{x y y}(\vec{r})$, and $\delta=h_{y y y}(\vec{r})$ are such that Eqs. (59) and (61) prescribe a monstar, given that the second derivatives obey $h_{x x}(\vec{r})=h_{y y}(\vec{r})$ and $h_{x y}(\vec{r})=0$.

In order to determine this, we require the joint probability distribution of these seven stochastic variables. When we have this, we can set $h_{x x}=h_{y y}$ and $h_{x y}=0$ and integrate $\alpha, \beta, \gamma$, and $\delta$ over the appropriate ranges to get the density of monstars and all umbilical points, respectively. The ratio of these then gives the monstar fraction.

The calculation is detailed in Appendix D. The final result is

$$
\alpha_{M}=0.053+0.429 \mu\left\langle f^{\prime \prime \prime}(H)\right\rangle+O\left(f^{2}\right),
$$

where

$$
\mu \equiv \frac{K_{4}^{3}}{K_{6}^{2}} \quad(0 \leqslant \mu \leqslant 1) .
$$

There is an alternative expression for the term $\left\langle f^{\prime \prime \prime}(H)\right\rangle$ in Eq. (62). Since $H$ is Gaussian, with mean 0 and deviation
$\sqrt{K_{0}}=1$, we can write

$$
\left\langle f^{\prime \prime \prime}(H)\right\rangle=\int d z f^{\prime \prime \prime}(z) e^{-z^{2} / 2} .
$$

Repeated partial integration yields

$$
\begin{aligned}
\left\langle f^{\prime \prime \prime}(H)\right\rangle & =\int d z z f^{\prime \prime}(z) e^{-z^{2} / 2} \\
& =\int d z\left(z^{2}-1\right) f^{\prime}(z) e^{-z^{2} / 2} \\
& =\int d z\left(z^{3}-3 z\right) f(z) e^{-z^{2} / 2} \\
& =\left\langle\left(H^{3}-3 H\right) f(H)\right\rangle .
\end{aligned}
$$

The kurtosis of a stochastic variable is defined as the fourth cumulant divided by the square of the second, which gives

$$
\kappa \equiv \frac{\left\langle h^{4}\right\rangle}{\left\langle h^{2}\right\rangle^{2}}-3 .
$$

If we enter $h=H+f(H)$, we find

$$
\begin{aligned}
\kappa & =\frac{\left\langle H^{4}\right\rangle+4\left\langle H^{3} f(H)\right\rangle}{\left\langle H^{2}\right\rangle+4\langle H f(H)\rangle}-3+O\left(f^{2}\right) \\
& =\frac{4\left\langle H^{3} f(H)\right\rangle-12\langle H f(H)\rangle}{1+4\langle H f(H)\rangle}+O\left(f^{2}\right) \\
& =4\left\langle H^{3} f(H)\right\rangle-12\langle H f(H)\rangle+O\left(f^{2}\right) .
\end{aligned}
$$

We see that $\left\langle f^{\prime \prime \prime}(H)\right\rangle=\kappa / 4$ up to first order, which remains true if $K_{0} \neq 1$. Hence, an alternative form of Eq. (62) is

$$
\alpha_{M}=0.053+0.107 \mu \kappa+O\left(f^{2}\right),
$$

where $\kappa$ is the kurtosis of $h$.

\section{B. Comparison with simulations}

The most basic example of a non-Gaussian variable for which Eq. (62) can be tested is $h=H+\varepsilon H^{3}$, for which $\left\langle f^{\prime \prime \prime}(H)\right\rangle=6 \varepsilon$. Then, we have

$$
\alpha_{M}=0.053+2.576 \mu \varepsilon+O\left(\varepsilon^{2}\right) .
$$

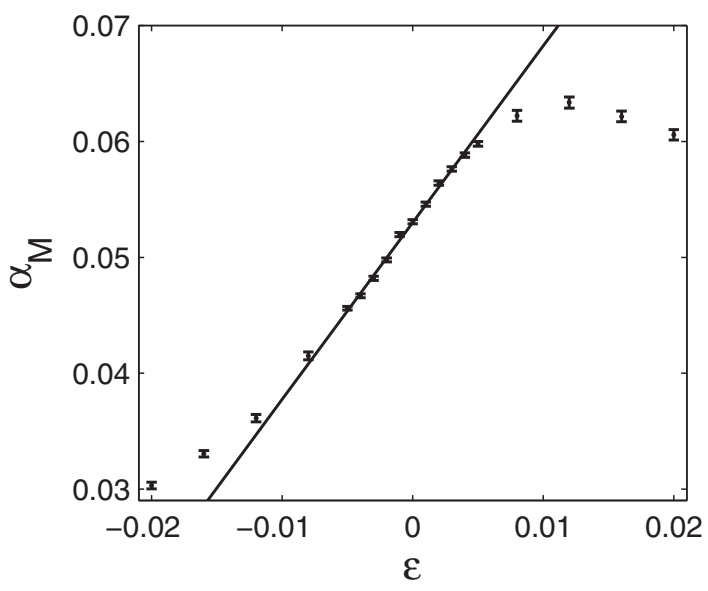

FIG. 8. The monstar fraction $\alpha_{M}$ of $H+\varepsilon H^{3}$ as a function of $\varepsilon$, where $H$ has a disk spectrum $\left(\mu=\frac{16}{27}\right)$. The data points stem from simulations, the solid line is Eq. (69). 


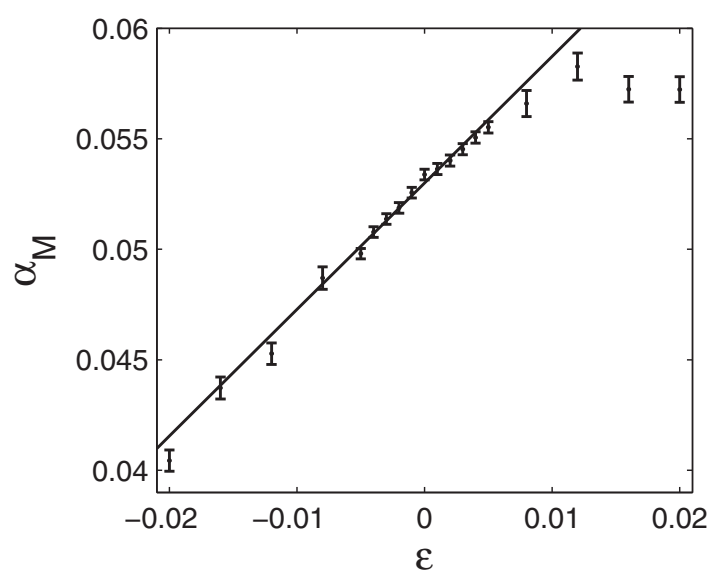

FIG. 9. The monstar fraction $\alpha_{M}$ of $H+\varepsilon H^{3}$ as a function of $\varepsilon$, where $H$ has a Gaussian spectrum $\left(\mu=\frac{2}{9}\right)$. The data points stem from simulations, the solid line is Eq. (69).

Equation (62) was compared to results from simulations: the procedure was analogous to the one outlined in Appendix $B$. We chose the same spectra as before: a disk spectrum

$$
A(k)^{2} \sim \theta\left(k_{0}-k\right), \quad K_{2 n}=\frac{k_{0}^{2 n}}{n+1}, \quad \mu=\frac{16}{27},
$$

and a Gaussian spectrum

$$
A(k)^{2} \sim \exp \left(-k^{2} / 2 k_{0}^{2}\right), \quad K_{2 n}=2^{n} n ! k_{0}^{2 n}, \quad \mu=\frac{2}{9} .
$$

A very good agreement between theory and simulation was found for both spectra (see Figs. 8 and 9), for $\varepsilon$ up to about 0.01 . For larger values of $\varepsilon$, nonlinear terms start to dominate.

Another thing to note is the sensitivity: In Eq. (69), we see that the prefactor of the perturbation term is very large compared to the leading order. As a result, even for small $\varepsilon$, the relative deviation from the universal 0.053 is quite large, as can be seen in the graphs. Therefore, measuring the monstar fraction of a given field proves to be a good method for detecting and quantifying small deviations from Gaussianity.

\section{CONCLUSIONS}

For a random field given by $h(\vec{r})=F_{N L}[H(\vec{r})]$, where $H$ is a Gaussian field and $F_{N L}$ any (nonlinear) function, we find that the densities of maxima and minima of $h$ may differ. We have shown what the imbalance is as a function of the transformation $F_{N L}$ and the power spectrum of $H$. Our result is exact, and does not rely on perturbation theory, a nice feature since $F_{N L}$ does not have to be small for our result to apply. This is confirmed by our simulations.

Furthermore, we have calculated how the density of monstars changes in Appendix D, in the case that $h=H+f(H)$, where $f$ is a small perturbation. Comparing our formula to data allows us to measure the parameter $\left\langle f^{\prime \prime \prime}(H)\right\rangle=\kappa / 4$ of the nonGaussian contribution. In the calculation we used cumulants to derive the probability distribution for the derivatives of $h$. Even though in general these have an infinite number of nonzero cumulants up to first order in $f$, it turns out that the cumulants of the variables which are relevant for the umbilics $\left(h_{z z}, h_{z z z}\right.$, and $h_{z z z^{*}}$ at a single point) vanish beyond the fourth order due to symmetry. As a result, we found the interesting result that (up to first order) $\alpha_{M}$ depends only on $\left\langle f^{\prime \prime \prime}(H)\right\rangle$.

For a more general type of non-Gaussian field, there would be more independent variables and hence more nonzero cumulants. However, it often still holds that the higher-order cumulants are of less importance. In this case, one can consider only the cumulants up to a specific order (e.g., fourth order), of which still many would be zero due to symmetry. Applying the same procedure as outlined here could then reveal the monstar fraction up to first order.

While the imbalance between maxima and minima requires a relatively large local perturbation to become nonzero, the monstar fraction is quite sensitive to small $\varepsilon$. Another striking difference between the two is seen from considering a perturbation of the form $h=H+\varepsilon H^{n}$, where $n>1$ is an integer. The maxima and minima only differ when $n$ is even since for odd $n$ the symmetry between $h$ and $-h$ is not broken. On the other hand, the result for the monstar fraction requires $n$ to be odd, otherwise $\left\langle f^{\prime \prime \prime}(H)\right\rangle=0$. The two tests of non-Gaussianity are thus truly independent measures.

\section{ACKNOWLEDGMENTS}

This work was supported by the Dutch Foundation for Fundamental Research on Matter (FOM), the Dutch Foundation for Scientific Research (NWO), and the European Research Council (ERC). We thank T. Lubensky, R. D. Kamien, B. Jain, A. Boyarsky, L. Mahadevan, B. Chen, and W. van Saarloos for stimulating discussions.

\section{APPENDIX A: PROPERTIES OF GAUSSIAN FIELDS}

Let us start by calculating the mean and standard deviation of a Gaussian field $H$, the equivalents of $\mu$ and $\sigma$ of a Gaussian variable. This involves expectation values, which are obtained by integrating over all possible values of all random variables, which in this case, are the uniformly distributed phases

$$
\langle\ldots\rangle \equiv\left(\prod_{\vec{k}} \int \frac{d \phi_{\vec{k}}}{2 \pi}\right) \ldots
$$

The mean is then simply

$$
\begin{aligned}
\langle H(\vec{r})\rangle & =\left\langle H_{0}+\sum_{\vec{k}} A(k) \cos \left(\vec{k} \cdot \vec{r}+\phi_{\vec{k}}\right)\right\rangle \\
& =H_{0}+\sum_{\vec{k}} A(k)\left\langle\cos \left(\vec{k} \cdot \vec{r}+\phi_{\vec{k}}\right)\right\rangle \\
& =H_{0}+\sum_{\vec{k}} A(k) \int \frac{d \phi_{\vec{k}}}{2 \pi} \cos \left(\vec{k} \cdot \vec{r}+\phi_{\vec{k}}\right)=H_{0} .
\end{aligned}
$$


For the variance (standard deviation squared) we find

$$
\begin{aligned}
\left\langle(H-\langle H\rangle)^{2}\right\rangle= & \left\langle\left(\sum_{\vec{k}} A(k) \cos \left(\vec{k} \cdot \vec{r}+\phi_{\vec{k}}\right)\right)^{2}\right\rangle \\
= & \sum_{\vec{k} \vec{k}^{\prime}} A(k) A\left(k^{\prime}\right)\left\langle\cos \left(\vec{k} \cdot \vec{r}+\phi_{\vec{k}}\right)\right. \\
& \left.\times \cos \left(\overrightarrow{k^{\prime}} \cdot \vec{r}+\phi_{\overrightarrow{k^{\prime}}}\right)\right\rangle .
\end{aligned}
$$

Since the phases are uncorrelated, for $\vec{k} \neq \overrightarrow{k^{\prime}}$ we find

$$
\begin{aligned}
& \left\langle\cos \left(\vec{k} \cdot \vec{r}+\phi_{\vec{k}}\right) \cos \left(\overrightarrow{k^{\prime}} \cdot \vec{r}+\phi_{\vec{k}^{\prime}}\right)\right\rangle \\
& \quad=\left\langle\cos \left(\vec{k} \cdot \vec{r}+\phi_{\vec{k}}\right)\right\rangle\left\langle\cos \left(\overrightarrow{k^{\prime}} \cdot \vec{r}+\phi_{\vec{k}^{\prime}}\right)\right\rangle=0 .
\end{aligned}
$$

Hence, the term in the double sum can only be nonzero for $\vec{k}=\vec{k}^{\prime}$. As a result, we get

$$
\begin{aligned}
\left\langle(H-\langle H\rangle)^{2}\right\rangle & =\sum_{\vec{k}} A(k)^{2}\left\langle\cos ^{2}\left(\vec{k} \cdot \vec{r}+\phi_{\vec{k}}\right)\right\rangle \\
& =\sum_{\vec{k}} \frac{1}{2} A(k)^{2} .
\end{aligned}
$$

For simplicity, we will set $\langle H\rangle=0$ and $\left\langle H^{2}\right\rangle=1$, which translates to $H_{0}=0$ and $\sum_{\vec{k}} \frac{1}{2} A(k)^{2}=1$.

While the vectors $\vec{k}$ in Eq. (3) form a discrete set, usually they are sufficiently finely spaced so that we can treat the amplitude spectrum $A(k)$ as a continuous function defined over the positive reals. If we take our normalization condition, and replace the sum with an integral, we get

$$
\begin{aligned}
1 & =\sum_{\vec{k}} \frac{1}{2} A(k)^{2}=\int d \vec{k} \frac{1}{2} a(k)^{2}=\int_{0}^{2 \pi} \int_{0}^{\infty} k d k d \theta \frac{1}{2} a(k)^{2} \\
& =\int_{0}^{\infty} d k \pi k a(k)^{2}=\int_{0}^{\infty} d k \Pi(k) .
\end{aligned}
$$

Here, $a(k)$ indicates the continuous spectrum equivalent to the discrete amplitudes $A(k)$. The newly introduced function $\Pi(k) \equiv \pi k a(k)^{2}$ is the power spectrum of $H$.

Some properties of a Gaussian field depend on the amplitude spectrum. In many cases, this dependence can be expressed in terms of the moments of the spectrum

$$
K_{n}=\sum_{\vec{k}} \frac{1}{2} A(k)^{2} k^{n}=\int_{0}^{\infty} d k \Pi(k) k^{n} .
$$

The normalization condition can be translated as $K_{0}=1$.

\section{Two-point correlation function}

Correlation functions are often used to probe the Gaussianity of a given random field. This is because for Gaussian fields, they obey certain relations, as reviewed below. We will first calculate the two-point correlation function. The two-point correlation function $C\left(\vec{r}_{1}, \vec{r}_{2}\right)$ of a field $\psi$ is defined as

$$
C\left(\vec{r}_{1}, \vec{r}_{2}\right)=\left\langle\psi\left(\vec{r}_{1}\right) \psi\left(\overrightarrow{r_{2}}\right)\right\rangle .
$$

When $\psi$ is homogeneous and isotropic, $C$ depends only on the distance between $\vec{r}_{1}$ and $\overrightarrow{r_{2}}$ :

$$
C(R)=\langle\psi(\vec{r}) \psi(\vec{r}+\vec{R})\rangle,
$$

where $\vec{r}$ is any position and $\vec{R}$ is any vector of length $R$. For a Gaussian field $H$ we find (if we set $\vec{r}=0$ for convenience, which we are free to do)

$$
\begin{aligned}
C(R) & =\langle H(0) H(\vec{R})\rangle \\
& =\left\langle\left(\sum_{\vec{k}} A(k) \cos \left(\phi_{\vec{k}}\right)\right)\left(\sum_{\vec{k}} A(k) \cos \left(\vec{k} \cdot \vec{R}+\phi_{\vec{k}}\right)\right)\right\rangle \\
& =\sum_{\vec{k} \vec{k}^{\prime}} A(k) A\left(k^{\prime}\right)\left\langle\cos \left(\phi_{\vec{k}}\right) \cos \left(\overrightarrow{k^{\prime}} \cdot \vec{R}+\phi_{\vec{k}^{\prime}}\right)\right\rangle .
\end{aligned}
$$

Since the phases $\phi_{\vec{k}}$ are uncorrelated, the correlation is automatically zero when $\vec{k} \neq \vec{k}^{\prime}$, hence

$$
\begin{aligned}
C(R) & =\sum_{\vec{k}} A(k)^{2}\left\langle\cos \left(\phi_{\vec{k}}\right) \cos \left(\vec{k} \cdot \vec{R}+\phi_{\vec{k}}\right)\right\rangle \\
& =\sum_{\vec{k}} A(k)^{2}\left\langle\frac{1}{2} \cos \left(\vec{k} \cdot \vec{R}+2 \phi_{\vec{k}}\right)+\frac{1}{2} \cos (\vec{k} \cdot \vec{R})\right\rangle .
\end{aligned}
$$

Since $\phi_{\vec{k}}$ is uniformly distributed, the expectation value of the first cosine is zero, and we are left with

$$
C(R)=\sum_{\vec{k}} \frac{1}{2} A(k)^{2} \cos (\vec{k} \cdot \vec{R})=\int d \vec{k} \frac{1}{2} a(k)^{2} \cos (\vec{k} \cdot \vec{R}) .
$$

We thus find that the two-point correlation function of a Gaussian field is the Fourier transform of its (two-dimensional) power spectrum. Therefore, in essence, the correlation function is as much a complete description of a Gaussian field as the power spectrum is. Also, by determining the correlation function and taking the inverse Fourier transform, one obtains the spectrum.

The moments, defined before in terms of the power spectrum, can be related to the derivatives of the correlation function at $R=0$. Because of symmetry, we must have $C(R)=C(-R)$. Hence, $C(R)$ is an even function and all its odd derivatives at zero vanish. To obtain the even derivatives, we must first eliminate the vector $\vec{R}$ in the equation above. We are free to choose its direction, so let us take $\vec{R}=R \hat{x}$. We then get

$$
\begin{aligned}
C^{(2 n)}(R) & =\left(\frac{d}{d R}\right)^{2 n} \int d \vec{k} \frac{1}{2} a(k)^{2} \cos \left(k_{x} R\right) \\
& =\int d \vec{k} \frac{1}{2} a(k)^{2}(-1)^{n} k_{x}^{2 n} \cos \left(k_{x} R\right), \quad(\mathrm{A} \\
C^{(2 n)}(0) & =(-1)^{n} \int d \vec{k} \frac{1}{2} a(k)^{2} k_{x}^{2 n} \\
& =(-1)^{n} \int_{0}^{2 \pi} \int_{0}^{\infty} k d k d \theta \frac{1}{2} a(k)^{2} k^{2 n} \cos ^{2 n} \theta \\
& =(-1)^{n} K_{2 n} \frac{1}{2 \pi} \int d \theta \cos ^{2 n} \theta \\
& =(-1)^{n} \frac{(2 n-1) ! !}{2^{n} n !} K_{2 n} .
\end{aligned}
$$

We thus find a one-to-one relation between the moments and the derivatives of the correlation function. The derivative of the correlation function $C^{(2 n)}(0)$ is related to roughness in the 
field itself. In fact, $C^{(2 n)}(0)$ is equal to $(-1)^{n}\left\langle\left[H^{(n)}(x)\right]^{2}\right\rangle$, the fluctuations of the $n$th derivative (apart from a sign).

\section{Higher-order correlation functions}

In general, the $n$-point correlation function is defined as the expectation value $\left\langle\psi\left(\vec{r}_{1}\right) \psi\left(\vec{r}_{2}\right) \ldots \psi\left(\vec{r}_{n}\right)\right\rangle$, as a function of $\overrightarrow{r_{1}}$ through $\overrightarrow{r_{n}}$. For a Gaussian field, this correlation function can be expressed in terms of two-point correlation functions, analogous to Wick's theorem. As a result, a non-Gaussian field can be recognized by checking whether this relation holds. In practice, this test can be applied to a single Gaussian field if it is homogeneous. In that case, the correlation function depends only on separations of the points $\overrightarrow{r_{2}}-\overrightarrow{r_{1}}$ through $\overrightarrow{r_{n}}-$ $\overrightarrow{r_{1}}$. From a given homogeneous field $\psi$, one obtains (a good approximation of) this correlation function by averaging over all (or a lot of) configurations with fixed spacings but translated to different $\overrightarrow{r_{1}}$ 's.

The simplest case of the relationship is

$$
\begin{aligned}
\left\langle H_{1} H_{2} H_{3} H_{4}\right\rangle= & \left\langle H_{1} H_{2}\right\rangle\left\langle H_{3} H_{4}\right\rangle+\left\langle H_{1} H_{3}\right\rangle\left\langle H_{2} H_{4}\right\rangle \\
& +\left\langle H_{1} H_{4}\right\rangle\left\langle H_{2} H_{3}\right\rangle,
\end{aligned}
$$

where we introduced the notation $H_{i} \equiv H\left(\vec{r}_{i}\right)$ for shortness. In general, correlations between an even number of variables with $n>2$ can be reduced to the two-point correlations, while correlations between an odd number of variables always vanish. These properties follow from the definition of the Gaussian field: the $n$ variables $H\left(r_{i}\right)$ are described by a correlated Gaussian distribution, and hence their correlation functions can be calculated explicitly from Gaussian integrals.

We shall now show how this characteristic relation comes about for our Fourier superposition. When we calculate the four-point correlation in the same way as we did for the twopoint correlation, we bring the brackets inside the (quadruple) sum, which gives us the term

$$
\begin{aligned}
& \left\langle\cos \left(\vec{k}_{1} \cdot \vec{r}_{1}+\phi_{\vec{k}_{1}}\right) \cos \left(\vec{k}_{2} \cdot \vec{r}_{2}+\phi_{\vec{k}_{2}}\right)\right. \\
& \left.\quad \times \cos \left(\vec{k}_{3} \cdot \vec{r}_{3}+\phi_{\vec{k}_{3}}\right) \cos \left(\vec{k}_{4} \cdot \vec{r}_{4}+\phi_{\vec{k}_{4}}\right)\right\rangle,
\end{aligned}
$$

which is summed for all combinations of $\vec{k}_{1}$ through $\vec{k}_{4}$. The first thing to note is that whenever, e.g., $\vec{k}_{1}$ is not equal to any of the other $\vec{k}_{i}$, the correlation is automatically zero; this is because $\cos \left(\vec{k}_{1} \cdot \vec{r}_{1}+\phi_{\vec{k}_{1}}\right)$ is then independent of all other factors, can therefore be separated, and gives zero. Hence, the correlation can only be nonzero if each $\vec{k}_{i}$ is equal to (at least) one other $\vec{k}_{i}$. We can distinguish the cases $\vec{k}_{1}=\vec{k}_{2}, \vec{k}_{3}=\vec{k}_{4}$ and $\vec{k}_{1}=\vec{k}_{3}, \vec{k}_{2}=\vec{k}_{4}$ and $\vec{k}_{1}=\vec{k}_{4}, \vec{k}_{2}=\vec{k}_{3}$. Let us focus on the first case; the sum of all these correlations gives

$$
\begin{aligned}
& \sum_{\overrightarrow{k_{1}}, \overrightarrow{k_{3}}} A\left(k_{1}\right)^{2} A\left(k_{3}\right)^{2}\left\langle\cos \left(\vec{k}_{1} \cdot \vec{r}_{1}+\phi_{\vec{k}_{1}}\right) \cos \left(\overrightarrow{k_{1}} \cdot \overrightarrow{r_{2}}+\phi_{\vec{k}_{1}}\right)\right. \\
& \left.\quad \times \cos \left(\overrightarrow{k_{3}} \cdot \overrightarrow{r_{3}}+\phi_{\vec{k}_{3}}\right) \cos \left(\overrightarrow{k_{3}} \cdot \overrightarrow{r_{4}}+\phi_{\vec{k}_{3}}\right)\right\rangle .
\end{aligned}
$$

This can be split into

$$
\begin{aligned}
\sum_{\overrightarrow{k_{1}}} A\left(k_{1}\right)^{2}\left\langle\cos \left(\vec{k}_{1} \cdot \vec{r}_{1}+\phi_{\vec{k}_{1}}\right) \cos \left(\vec{k}_{1} \cdot \overrightarrow{r_{2}}+\phi_{\vec{k}_{1}}\right)\right\rangle \\
\times \sum_{\overrightarrow{k_{3}}} A\left(k_{3}\right)^{2}\left\langle\cos \left(\vec{k}_{3} \cdot \vec{r}_{3}+\phi_{\vec{k}_{3}}\right) \cos \left(\vec{k}_{3} \cdot \vec{r}_{4}+\phi_{\vec{k}_{3}}\right)\right\rangle \\
\quad=\left\langle H\left(\overrightarrow{r_{1}}\right) H\left(\overrightarrow{r_{2}}\right)\right\rangle\left\langle H\left(\vec{r}_{3}\right) H\left(\vec{r}_{4}\right)\right\rangle .
\end{aligned}
$$

Applying the same to the other cases and adding them together precisely gives Eq. (A15).

One may note that the case $\vec{k}_{1}=\vec{k}_{2}=\vec{k}_{3}=\vec{k}_{4}$ has not been treated correctly. However, since $\vec{k}_{i}$ can take on an infinite number of values, and this case only provides one degree of freedom instead of the two we had for the other cases, these correlations only have an infinitesimal contribution.

From this example, it is not hard to see that in general, an $n$-point correlation function can be factorized, that is, written as the sum of products of two-point correlations, where the sum features all possible ways in which the $n$ variables can be paired up.

\section{APPENDIX B: COMPUTER SIMULATIONS}

In order to verify our theoretical results, we use a large number of computer-generated realizations of the Gaussian field $H$ (typically a few thousand), each with the same spectrum $A(k)$ but random phases $\phi_{\vec{k}}$. We then apply the desired transformation $F_{N L}$ and extract the desired statistics.

The fields are defined on a square with periodic boundary conditions, i.e., $H(x, y)=H(x+L, y)=H(x, y+L)$, in order to reduce finite size effects. This is accomplished by only adding together waves [as in Eq. (3)] with wave vectors $\vec{k}$ of which the $x$ and $y$ components are multiples of $2 \pi / L$.

The summation in Eq. (3) is restricted to wave vectors with a magnitude below a certain threshold $k_{\max }$. In order to minimize the potential effects of this cutoff, we choose spectra for which $A(k)$ decays very quickly or is zero for large $k$, such as the disk spectrum

$$
A(k)^{2} \sim \theta\left(k_{0}-k\right),
$$

and the Gaussian spectrum

$$
A(k)^{2} \sim \exp \left(-k^{2} / 2 k_{0}^{2}\right) .
$$

Finally, $L$ is chosen in relation to $k_{\max }$ such that (1) the sum in Eq. (3) features at least a few hundred waves (recall that $L$ influences this number via the periodic boundary conditions) and that (2) $L$ is at least a few times $2 \pi / \sqrt{K_{2}}$, which is a measure of the typical wavelength of the spectrum. An example of a Gaussian field generated in this way is shown in Fig. 1.

The resulting formula for $H$ is then evaluated at the grid points only. The distance between neighboring grid points is taken to be much smaller (by a factor of 50 roughly) than the typical wavelength. Along with $H$ itself, we also calculate its first, second, and third derivatives at these grid points.

We use a very efficient approach to identifying critical and umbilical points and their type. We will focus on the critical points to illustrate the process; the umbilics are identified in similar fashion. Every square of four neighboring grid points is considered. If $H_{x}$ or $H_{y}$ has the same sign at all four points, we infer that it is not zero anywhere inside the square, which leads to the conclusion that the square does not contain a critical point. Otherwise, there would necessarily be at least two pairs of neighboring grid points with a different sign of $H_{x}$. For each pair, it is assumed that $H_{x}$ changes linearly between the two points, which allows us to pinpoint two points along the edges of the square where $H_{x}=0$. The contour line $H_{x}=0$ is then assumed to be a straight line between these two points. The same recipe is applied to $H_{y}$, after which 


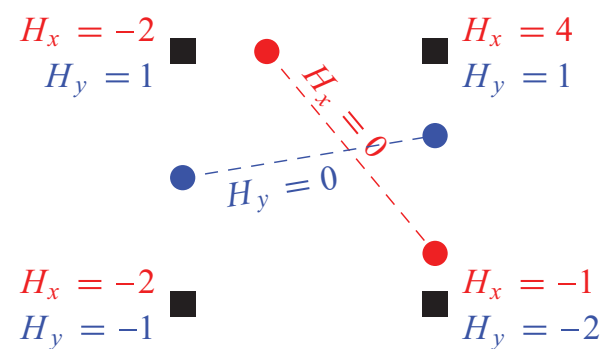

FIG. 10. (Color online) Identifying a critical point. The four black squares are grid points at which $H_{x}$ and $H_{y}$ are known. At the red (blue) dots, $H_{x}=0\left(H_{y}=0\right)$ under the assumption that $H_{x}\left(H_{y}\right)$ is linear between the two grid points. The two contour lines $H_{x}=0$ and $H_{y}=0$ then intersect inside the square, indicating the existence of a critical point.

it is determined whether the two contour lines crossed. The intersection (if present) is then a critical point. This idea is illustrated in Fig. 10.

It is also possible for all four neighboring points to have opposite signs of $H_{x}$ (or $H_{y}$ ). This results in four points along the border of the square with $H_{x}=0$, but without any information about which two pairs should be connected by a contour line. In combination with the two points with $H_{y}=0$ it can, however, be established what the parity of the number of intersections (i.e., critical points) is. We then simply assume this number to be 0 or 1 . This case is sufficiently rare (provided the grid is small enough) to not have a noticeable effect on the results.

Once established whether the square under consideration contains a critical point, the type is determined by averaging the values of $H_{x x}, H_{y y}$, and $H_{x y}$ at the four grid points and evaluating the signs of $H_{x x} H_{y y}-H_{x y}^{2}$ and $H_{x x}+H_{y y}$. Although this method clearly does not always correctly determine the existence of a critical point or its type, it is not biased toward one outcome. Therefore, the mistakes that are made will get averaged out when statistics are taken over a large number of critical points. With regard to getting good statistics, the speed of this method is a big advantage.

This method, along with the proper values of $k_{\max }, L$, and the grid size, was thoroughly tested on Gaussian fields (for which the statistical outcomes are known from theory) to verify its validity, before applying it to the non-Gaussian fields under investigation.

\section{APPENDIX C: ASYMPTOTES FOR A VERY SMALL NON-GAUSSIANITY}

In the limit where $z$ is very large and negative, $g(z)$ can be evaluated asymptotically. One can use the exact expression for $g(z)$, but returning to the original integral (35) gives more insight (and makes the calculations shorter). The exponential weight in the integral is peaked at $s=\frac{1}{2}\left(H_{x x}+H_{y y}\right)=-\frac{K_{2} z}{2}$. Therefore, $s$ almost certainly becomes large and positive when $-z$ is large since the width of the distribution remains fixed. This allows us to extend the range of integration to all $s$ 's and to all $r \geqslant 0$ since the additional parts of the range have a very small weight. Then, the integral can be worked out exactly, giving $g(z) \approx \sqrt{\frac{6}{\pi}} \lambda z^{2} e^{-\frac{z^{2}}{2}}$ apart from small corrections, when $-z$ is large and positive. We next substitute back in Eq. (8). Noting that $P$ dominates over $Q$ and using integration by parts to evaluate the integral asymptotically $\left(\int_{A}^{\infty} z^{2} e^{-\frac{z^{2}}{2}} d x \approx\right.$ $A e^{-\frac{A^{2}}{2}}$ when $\left.A \rightarrow \infty\right)$ gives $\Delta n=\sqrt{\frac{3}{2 \pi} \frac{\lambda}{\varepsilon}} e^{-\frac{1}{8 \varepsilon^{2}}}$.

\section{APPENDIX D: MONSTAR FRACTION: CALCULATION}

We consider a field of the form $h(\vec{r})=H(\vec{r})+f[H(\vec{r})]$, where $H(\vec{r})$ is a Gaussian field and $f$ a small nonlinear function of $H(\vec{r})$ only. The monstar fraction is the density of the monstars divided by the total density of umbilical points. An umbilical point is defined by Eq. (54), while the type of umbilical point (lemon, monstar, or star) can be determined by Eqs. (59) and (61). These equations involve the second and third derivatives of the field $h$.

We consider an arbitrary point $\vec{r}$. Note that due to the homogeneity of $H$ and $h$, the following analysis does not depend on the choice of $\vec{r}$. The monstar fraction is equal to the probability that $\vec{r}$ is a monstar, given that it is an umbilical point. This can be calculated from the joint probability distribution of the values of the derivatives of $h$ at this point.

If $h$ were a Gaussian field, then these derivatives would form a set of correlated Gaussian random variables. We can exploit the near-Gaussianity of $h$ to find the probability distribution perturbatively. This is achieved by determining the corresponding characteristic function, which is defined as the Fourier transform of the probability distribution [29].

\section{Characteristic function}

For a set of $n$ correlated variables $\left\{h_{i}\right\}$, the characteristic function of their joint probability distribution $p$ is defined as

$$
\begin{aligned}
\chi & \left(\lambda_{1}, \ldots, \lambda_{n}\right) \\
= & \int d h_{1} \ldots d h_{n} p\left(h_{1}, \ldots, h_{n}\right) e^{i\left(h_{1} \lambda_{1}+\cdots+h_{n} \lambda_{n}\right)} \\
= & 1+i \sum_{j}\left\langle h_{j}\right\rangle \lambda_{j}+\frac{i^{2}}{2 !} \sum_{j_{1}, j_{2}}\left\langle h_{j_{1}} h_{j_{2}}\right\rangle \lambda_{j_{1}} \lambda_{j_{2}} \\
& +\frac{i^{3}}{3 !} \sum_{j_{1}, j_{2}, j_{3}}\left\langle h_{j_{1}} h_{j_{2}} h_{j_{3}}\right\rangle \lambda_{j_{1}} \lambda_{j_{2}} \lambda_{j_{3}}+\cdots .
\end{aligned}
$$

Here, the coefficients $\langle\ldots\rangle$ are the moments, or multivariable correlations, defined by

$$
\left\langle h_{j_{1}} \ldots h_{j_{k}}\right\rangle \equiv \int d h_{1} \ldots d h_{n} p\left(h_{1}, \ldots, h_{n}\right) h_{j_{1}} \ldots h_{j_{k}} .
$$

Equation (D1) is proved by expanding the exponential term by term.

Upon taking the logarithm of $\chi$ and expanding, the quantities known as the cumulants are revealed:

$$
\begin{aligned}
\ln \chi= & i \sum_{j} C_{1}\left(h_{j}\right) \lambda_{j}+\frac{i^{2}}{2 !} \sum_{j_{1}, j_{2}} C_{2}\left(h_{j_{1}}, h_{j_{2}}\right) \lambda_{j_{1}} \lambda_{j_{2}} \\
& +\frac{i^{3}}{3 !} \sum_{j_{1}, j_{2}, j_{3}} C_{3}\left(h_{j_{1}}, h_{j_{2}}, h_{j_{3}}\right) \lambda_{j_{1}} \lambda_{j_{2}} \lambda_{j_{3}}+\cdots .
\end{aligned}
$$

The cumulants can be written in terms of the moments, as can be seen by taking the logarithm of Eq. (D1) and expanding it. 
For example,

$$
\begin{aligned}
C\left(h_{1}, h_{2}, h_{3}\right)= & \left\langle h_{1} h_{2} h_{3}\right\rangle-\left\langle h_{1}\right\rangle\left\langle h_{2} h_{3}\right\rangle-\left\langle h_{2}\right\rangle\left\langle h_{3} h_{1}\right\rangle \\
& -\left\langle h_{3}\right\rangle\left\langle h_{1} h_{2}\right\rangle+2\left\langle h_{1}\right\rangle\left\langle h_{2}\right\rangle\left\langle h_{3}\right\rangle .
\end{aligned}
$$

In reverse, the moments can be written in terms of the cumulants, e.g.,

$$
\begin{aligned}
\left\langle h_{1} h_{2} h_{3}\right\rangle= & C\left(h_{1}, h_{2}, h_{3}\right)+C\left(h_{1}\right) C\left(h_{2}, h_{3}\right)+C\left(h_{2}\right) C\left(h_{3}, h_{1}\right) \\
& +C\left(h_{3}\right) C\left(h_{1}, h_{2}\right)+C\left(h_{1}\right) C\left(h_{2}\right) C\left(h_{3}\right) . \quad(\mathrm{D} 5)
\end{aligned}
$$

If all the moments or all the cumulants are known, we can construct the characteristic function and perform an inverse Fourier transformation to obtain the probability distribution.

The defining characteristic of Gaussian random variables $H_{i}$ is that all cumulants are zero, with the exception of the second-order ones $C_{2}\left(H_{i}, H_{j}\right)=\left\langle H_{i} H_{j}\right\rangle$. In this case, the characteristic function is thus

$$
\chi\left(\lambda_{1}, \ldots, \lambda_{n}\right)=\exp \left(-\frac{1}{2} \sum_{i j} C_{2}\left(H_{i}, H_{j}\right) \lambda_{i} \lambda_{j}\right) .
$$

The inverse Fourier transformation yields the standard distribution for correlated Gaussian random variables Eq. (12).

For a Gaussian field $H$, the derivatives are themselves Gaussian fields; therefore, the above formula gives their joint distribution. For the non-Gaussian field $h$, there are some small corrections to this distribution. To find these corrections to first order, we need to determine the cumulants to first order in $f(H)$. We will see that only a small number of cumulants are nonzero up to this order. Before we proceed to derive them, we switch to a complex coordinate system which allows for optimal usage of translational and rotational symmetry, which $h$ has inherited from $H$ for the type of perturbations under consideration.

\section{Complex coordinates representation}

To find the distribution of umbilical points, we now have to find the joint distribution of the seven second and third derivatives of $h$. All these variables can be combined into a more compact form by using complex coordinates. These will make it easier to evaluate the integral that determines the monstar density, and will help us to work out the probability distribution with the help of symmetry.

The complex coordinates are given by

$$
\begin{aligned}
z & =x+i y, \quad x=\frac{1}{2}\left(z+z^{*}\right), \\
z^{*}=x-i y, \quad y & =\frac{1}{2} i\left(z^{*}-z\right) .
\end{aligned}
$$

Of course, as complex numbers, $z$ and $z^{*}$ are not independent; however, we can formally define partial derivatives with respect to each of them, using the chain rule, just like we could if this transformation involved a real number instead of $i$.

The derivatives with respect to $z$ and $z^{*}$ are given by

$$
\frac{\partial}{\partial z}=\frac{1}{2} \frac{\partial}{\partial x}-\frac{1}{2} i \frac{\partial}{\partial y}, \quad \frac{\partial}{\partial z^{*}}=\frac{1}{2} \frac{\partial}{\partial x}+\frac{1}{2} i \frac{\partial}{\partial y} .
$$

We see that the derivatives with respect to $z$ and $z^{*}$ are each other's conjugate, but again, we consider both to be linear transformations of $\partial_{x}$ and $\partial_{y}$. The usefulness of using $z$ and $z^{*}$ can be immediately seen from $h_{z z}$ :

$$
h_{z z}=\partial_{z}^{2} h=\frac{1}{4}\left(\partial_{x}-i \partial_{y}\right)^{2} h=\frac{1}{4}\left(h_{x x}-h_{y y}+2 i h_{x y}\right) .
$$

We see that the definition of an umbilical point can be captured in one equation: $h_{z z}=0$.

The various types of umbilical points were defined in Eqs. (59) and (61) using the "normal" third derivatives $h_{x x x}=$ $\alpha, h_{x x y}=\beta, h_{x y y}=\gamma$, and $h_{y y y}=\delta$. In terms of $h_{z z z}, h_{z z z^{*}}$, $h_{z z^{*} z^{*}}$, and $h_{z^{*} z^{*} z^{*}}$ the two conditions for a monstar become

$$
\begin{aligned}
& \left|h_{z z z^{*}}\right|^{2}-\left|h_{z z z}\right|^{2}>0, \\
& 27\left|h_{z z z}\right|^{4}-\left|h_{z z z^{*}}\right|^{4}-18\left|h_{z z z}\right|^{2}\left|h_{z z z^{*}}\right|^{2} \\
& \quad-4\left(h_{z z z} h_{z z^{*} z^{*}}^{3}+h_{z^{*} z^{*} z^{*}} h_{z z z^{*}}^{3}\right)>0 .
\end{aligned}
$$

Here $\left|h_{z z z}\right|^{2}$ and $\left|h_{z z z^{*}}\right|^{2}$ represent $h_{z z z} h_{z^{*} z^{*} z^{*}}$ and $h_{z z z^{*}} h_{z z^{*} z^{*}}$, respectively.

The density of monstars will essentially be given by integrating the probability distribution $p\left(h_{z z}=0, h_{z z z}, h_{z z z^{*}}\right)$ over the range defined by these conditions. This probability distribution is determined by the cumulants of combinations of the three variables. Rotational and translational symmetry, however, imply that only a small number of these combinations yield a nonzero cumulant.

First, consider the consequences of the isotropy (rotational symmetry) of the field $h(\vec{r})$ for a moment like $\left\langle h_{z^{*}}(\vec{r}) h_{z z}(\vec{r})\right\rangle$. Note that, due to homogeneity (translational symmetry), this moment does not depend on $r$; it will often be dropped from now on. Isotropy implies that this moment should not change if we rotate the field around $\vec{r}$, over any angle $\alpha$. In terms of $z$ and $z^{*}$, this results in the transformation

$$
\begin{aligned}
z^{\prime} & =e^{i \alpha} z, \quad \partial_{z^{\prime}}=e^{-i \alpha} \partial_{z}, \\
z^{*} & =e^{-i \alpha} z^{*}, \quad \partial_{z^{*}}=e^{i \alpha} \partial_{z^{*}} .
\end{aligned}
$$

As a result, we get $\left\langle h_{z^{\prime}} h_{z^{* *} z^{* *}}\right\rangle=e^{i \alpha}\left\langle h_{z} h_{z^{*} z^{*}}\right\rangle$. Since we argued that the two expectation values must be equal, for any $\alpha$, we must have $\left\langle h_{z^{*}} h_{z z}\right\rangle=0$.

In general, following a rotation expectation values pick up a factor $e^{i k \alpha}$, where $k$ is the number of $z^{*}$ derivatives inside the bracket minus the number of $z$ derivatives. By the above argument, the expectation value is zero if $k \neq 0$. Therefore, an expectation value can only be nonzero if the numbers of $z$ and $z^{*}$ derivatives inside the bracket are equal. Since a cumulant is a sum of products of expectation values, featuring every variable once in every product [compare Eq. (D4)], the same property applies to cumulants.

The homogeneity (translational symmetry) of the fields under consideration provides another useful trick that relates different cumulants to one another. As already stated, a moment like $\left\langle h_{1}(\vec{r}) \ldots h_{n}(\vec{r})\right\rangle$ does not depend on $\vec{r}$. Hence, the derivative of this with respect to $z$ or $z^{*}$ is zero. Applying the product rule

$$
\begin{aligned}
0 & =\partial_{z}\left\langle h_{1} \ldots h_{n}\right\rangle \\
& =\left\langle\left(\partial_{z} h_{1}\right) h_{2} \ldots h_{n}\right\rangle+\cdots+\left\langle h_{1} h_{2} \ldots\left(\partial_{z} h_{n}\right)\right\rangle .
\end{aligned}
$$

For $n=2$, this gives the useful relation

$$
\left\langle\left(\partial_{z} h_{1}\right) h_{2}\right\rangle=-\left\langle h_{1}\left(\partial_{z} h_{2}\right)\right\rangle .
$$


In essence, for a two-point correlation it is possible to "transfer" a $z$ derivative from the one term to the other at the cost of an overall minus sign. The same applies of course to a $z^{*}$ derivative. For example, we find the relation $\left\langle h_{z z} h_{z^{*} z^{*}}\right\rangle=-\left\langle h_{z} h_{z z^{*} z^{*}}\right\rangle$.

Together, these two symmetries constrain the probability distribution $p\left(h_{z z}, h_{z z z}, h_{z z z^{*}}\right)$. In particular, they explain why the monstar fraction is always the same for any Gaussian distribution [7]. A Gaussian distribution does not have many degrees of freedom to start with; only the two-point correlations between the variables are adjustable. In this case, the two-point correlations between any two of these variables is zero, by rotational symmetry, while the variances of $h_{z z z}$ and $h_{z z z^{*}}$ are equal by translational symmetry. Hence (after setting $h_{z z}=0$ to identify the umbilical points), the distribution $p\left(h_{z z}=0, h_{z z z}, h_{z z z^{*}}\right)$ is always the same apart from a scale, and that determines the monstar fraction. This argument can be generalized to singularities in the polarization field of light (even though the field might not be derived from a scalar field $h$ ), and so Gaussian polarization fields have the same monstar fraction as well, as shown in [16].

On the other hand, when $h$ has non-Gaussian contributions, there are many more cumulants, and symmetry is not enough to constrain them any more. For the field $h=H+f(H)$ we are studying, we proceed to calculate the cumulants explicitly.

\section{Cumulants}

Although the problem is now cast in terms of complex derivatives, the recipe outlined in Sec. D 1 still applies. The task is to determine the cumulants of $h_{z z}, h_{z z z}, h_{z z z^{*}}$ and their conjugates up to first order in the perturbation $f$. These cumulants have the general form $C_{n}\left(D_{1} h, \ldots, D_{n} h\right)$, where each $D_{j}$ represents a number of $z$ and $z^{*}$ derivatives. For the moment, let us consider each $D_{j} h$ to be at a different point $\vec{r}_{j}$, i.e., $C_{n}\left(D_{1} h\left(\vec{r}_{1}\right), \ldots, D_{n} h\left(\vec{r}_{n}\right)\right)$. Later, we will set all points equal again. For convenience, we shall drop the vector notation, i.e., $r_{i}=\vec{r}_{i}$. Since now each derivative $D_{j}$ acts only at a specific point, we can bring them outside the cumulant:

$$
\begin{aligned}
& C_{n}\left(D_{1} h, \ldots, D_{n} h\right) \\
& \quad=\left.D_{1} \ldots D_{n} C_{n}\left(h\left(r_{1}\right), \ldots, h\left(r_{n}\right)\right)\right|_{r_{1}=\cdots=r_{n}} .
\end{aligned}
$$

Let us write $h\left(r_{j}\right)=h_{j}$ for shortness, and focus on $C_{n}\left(h_{1}, \ldots, h_{n}\right)$. Inserting $h_{j}=H_{j}+f\left(H_{j}\right)$, expanding the cumulant, and keeping only terms up to first order in $f$ yields

$$
\begin{aligned}
C_{n}\left(h_{1}, \ldots, h_{n}\right)= & C_{n}\left(H_{1}, \ldots, H_{n}\right) \\
& +C_{n}\left(f\left(H_{1}\right), H_{2}, \ldots, H_{n}\right) \\
& +C_{n}\left(H_{1}, f\left(H_{2}\right), \ldots, H_{n}\right)+\cdots y \\
& +C_{n}\left(H_{1}, H_{2}, \ldots, f\left(H_{n}\right)\right) .
\end{aligned}
$$

The first term on the right-hand side is now simply the cumulant of a set of Gaussian random variables, which, as discussed before, is zero for $n>2$. The other terms can be evaluated perturbatively and are equivalent to each other. Consider the second term as an example. For a cumulant involving Gaussian variables and one function of a Gaussian, we have (see Appendix E)

$$
\begin{aligned}
& C_{n}\left(f\left(H_{1}\right), H_{2}, \ldots, H_{n}\right) \\
& \quad=\left\langle f^{(n-1)}\left(H_{1}\right)\right\rangle\left\langle H_{1} H_{2}\right\rangle\left\langle H_{1} H_{3}\right\rangle \ldots\left\langle H_{1} H_{n}\right\rangle .
\end{aligned}
$$

When we reinsert the derivatives $D_{2}$ through $D_{n}$ from Eq. (D14) and set $r_{2}=\cdots=r_{n}=r$, we get

$$
\begin{aligned}
& \left.D_{1} \ldots D_{n} C_{n}\left(f\left[H\left(r_{1}\right)\right], \ldots, H\left(r_{n}\right)\right)\right|_{r_{1}=\cdots=r_{n}} \\
& \quad=\left.D_{1}\left\langle f^{(n-1)}\left(H_{1}\right)\right\rangle\left\langle H_{1} D_{2} H\right\rangle \ldots\left\langle H_{1} D_{n} H\right\rangle\right|_{r_{1}=r} .
\end{aligned}
$$

Now we can reinsert $D_{1}$ and then set $r_{1}=r$, as prescribed by Eq. (D14). Remember that $D_{1}$ only acts on $H_{1}$. Due to the product rule, we have to consider all possible ways in which the derivatives in $D_{1}$ can be distributed over all $H_{1}$ 's. Recall from Sec. D 2 that, after setting $r_{1}=r$, each expectation value can only be nonzero if the number of $z$ and $z^{*}$ derivatives inside are equal. Note also that $\left\langle f^{(n-1)}\left(H_{1}\right)\right\rangle$ does not depend on $r_{1}$, hence any derivative of it is zero. Therefore, the only nonzero contributions stemming from the product rule are those distributions that make the number of $z$ and $z^{*}$ derivatives equal inside each bracket.

We consider the cumulant $C_{3}\left(h_{z z}, h_{z z^{*} z^{*}}, h_{z z^{*} z^{*}}\right)$ as an example to demonstrate the procedure. First, we find

$$
\begin{aligned}
C_{3}( & \left.h_{z z}, h_{z z^{*} z^{*}}, h_{z z^{*} z^{*}}\right) \\
= & C_{3}\left(H_{z z}, H_{z z^{*} z^{*}}, H_{z z^{*} z^{*}}\right) \\
& +\partial_{z_{1} z_{1}}\left(\left\langle f^{\prime \prime}(H)\right\rangle\left\langle H_{1} H_{z z^{*} z^{*}}\right\rangle\left\langle H_{1} H_{z z^{*} z^{*}}\right\rangle\right) \\
& +2 \partial_{z_{2} z_{2}^{*} z_{2}^{*}}\left(\left\langle f^{\prime \prime}(H)\right\rangle\left\langle H_{2} H_{z z}\right\rangle\left\langle H_{2} H_{z z^{*} z^{*}}\right\rangle\right) .
\end{aligned}
$$

The first term is zero since all cumulants of Gaussian variables are zero beyond second order. For the second term, we need to consider how to distribute the two $\partial_{z_{1}}$ derivatives to make all expectation values nonzero. The only possibility is to put one $\partial_{z_{1}}$ in front of each $H_{1}$. Note, however, that this term appears twice in the product rule because there are two ways of distributing the two derivatives. After setting $r_{1}=r$ we thus have

$$
\begin{aligned}
& \partial_{z_{1} z_{1}}\left[\left\langle f^{\prime \prime}(H)\right\rangle\left\langle H_{1} H_{z z^{*} z^{*}}\right\rangle\left\langle H_{1} H_{z z^{*} z^{*}}\right\rangle\right] \\
& =2\left\langle f^{\prime \prime}(H)\right\rangle\left\langle H_{z} H_{z z^{*} z^{*}}\right\rangle^{2} .
\end{aligned}
$$

In the third term on the right-hand side of Eq. (D19) we have one $\partial_{z}$ and two $\partial_{z^{*}}$ 's to distribute. The first $H_{2}$ needs $\partial_{z^{*} z^{*}}$ to balance the derivatives and the other takes the $\partial_{z}$ derivative. There are no multiple ways to distribute these derivatives in this case and therefore

$$
\begin{gathered}
\partial_{z_{2} z_{2}^{*} z_{2}^{*}}\left[\left\langle f^{\prime \prime}(H)\right\rangle\left\langle H_{2} H_{z z}\right\rangle\left\langle H_{2} H_{z z^{*} z^{*}}\right\rangle\right] \\
=\left\langle f^{\prime \prime}(H)\right\rangle\left\langle H_{z^{*} z^{*}} H_{z z}\right\rangle\left\langle H_{z} H_{z z^{*} z^{*}}\right\rangle .
\end{gathered}
$$

Combining everything together results in

$$
\begin{aligned}
& C_{3}\left(h_{z z}, h_{z z^{*} z^{*}}, h_{z z^{*} z^{*}}\right) \\
& \quad=2\left\langle f^{\prime \prime}(H)\right\rangle\left\langle H_{z} H_{z z^{*} z^{*}}\right\rangle\left(\left\langle H_{z} H_{z z^{*} z^{*}}\right\rangle+\left\langle H_{z^{*} z^{*}} H_{z z}\right\rangle\right) .
\end{aligned}
$$

Finally, due to translational symmetry, we have $\left\langle H_{z} H_{z z^{*} z^{*}}\right\rangle+\left\langle H_{z^{*} z^{*}} H_{z z}\right\rangle=\partial_{z}\left\langle H_{z} H_{z^{*} z^{*}}\right\rangle=0$. We thus find that $C_{3}\left(h_{z z}, h_{z z^{*} z^{*}}, h_{z z^{*} z^{*}}\right)=0$.

Now, we will show that there are only a finite number of nonzero cumulants (up to first order in $f$ ). In fact, there are none beyond the fourth order. Consider Eq. (D15) with $n>4$. The first term (zero order) is zero because it is the cumulant of 
TABLE I. All nonzero cumulants. The two asymmetric cumulants have a conjugate twin in which all $z$ 's and $z^{*}$ 's are interchanged.

\begin{tabular}{lc}
\hline \hline$C_{2}\left(h_{z z}, h_{z^{*} z^{*}}\right)$ & $=\sigma\left[1+2\left\langle f^{\prime}(H)\right\rangle\right]$ \\
$C_{2}\left(h_{z z z}, h_{z^{*} z^{*} z^{*}}\right)$ & $=\tau\left[1+2\left\langle f^{\prime}(H)\right\rangle\right]$ \\
$C_{2}\left(h_{z z z^{*}}, h_{z z^{*} z^{*}}\right)$ & $=\tau\left[1+2\left\langle f^{\prime}(H)\right\rangle\right]$ \\
$C_{3}\left(h_{z z}, h_{z z z^{*}}, h_{z^{*} z^{*} z^{*}}\right)+$ conj. & $=-3 \sigma^{2}\left\langle f^{\prime \prime}(H)\right\rangle$ \\
$C_{4}\left(h_{z z z^{*}}, h_{z z z^{*}}, h_{z z^{*} z^{*}}, h_{z z^{*} z^{*}}\right)$ & $=-8 \sigma^{3}\left\langle f^{\prime \prime \prime}(H)\right\rangle$ \\
$C_{4}\left(h_{z z z}, h_{z z^{*} z^{*}}, h_{z z^{*} z^{*}}, h_{z z^{*} z^{*}}\right)+$ conj. & $=-6 \sigma^{3}\left\langle f^{\prime \prime \prime}(H)\right\rangle$ \\
\hline \hline
\end{tabular}

more than two Gaussian variables. For the other ones we apply the recipe of Eq. (D17). We have $n-1$ brackets in which the $z$ and $z^{*}$ derivatives need to be matched. Since we are only considering the variables $h_{z z}, h_{z z z}, h_{z z z^{*}}$, and their conjugates, each one has a mismatch to begin with. However, since $D_{1}$ has only three derivatives at most, it is not possible to balance the derivatives in all $n-1$ brackets.

This "lack of derivatives" also kills a lot of cumulants of lower order, especially fourth order. For example, in $C_{4}\left(h_{z z}, h_{z^{*} z^{*}}, h_{z z z^{*}}, h_{z z^{*} z^{*}}\right)$ the first two variables require two derivatives to balance the derivatives and the other two require one. Therefore, no matter from which variable the derivatives are distributed, there is always a shortage.

All the nonzero cumulants are listed in Table I. Two parameters were introduced:

$$
\begin{aligned}
\sigma & \equiv\left\langle H_{z z} H_{z^{*} z^{*}}\right\rangle=-\left\langle H_{z} H_{z z^{*} z^{*}}\right\rangle, \\
\tau & \equiv\left\langle H_{z z z} H_{z^{*} z^{*} z^{*}}\right\rangle=\left\langle H_{z z z^{*}} H_{z z^{*} z^{*}}\right\rangle .
\end{aligned}
$$

Note that the trick based on translational symmetry was used to equate the expectation values. In the second equation, it was used twice (transferring a $\partial_{z}$ one way and a $\partial_{z^{*}}$ the other way).

The parameters $\sigma$ and $\tau$ are related to the moments $K_{n}$ of $H$. This is most easily accomplished by writing $H$ in complex variables:

$$
\begin{aligned}
H & =\sum_{\vec{k}} A(k) \cos \left(\vec{k} \cdot \vec{r}+\phi_{\vec{k}}\right) \\
& =\sum_{k} A(|k|) \cos \left[\frac{1}{2}\left(k^{*} z+k z^{*}\right)+\phi_{k}\right] .
\end{aligned}
$$

Here, $k=k_{x}+i k_{y}$ is the complex analog of $\vec{k}=\left(\begin{array}{c}k_{x} \\ k_{y}\end{array}\right)$. With this, we find

$$
\begin{aligned}
H_{z z z} & =\sum_{k} A(k) \frac{1}{8}\left(k^{*}\right)^{3} \sin \left[\frac{1}{2}\left(k^{*} z+k z^{*}\right)+\phi_{k}\right], \\
H_{z^{*} z^{*} z^{*}} & =\sum_{k} A(k) \frac{1}{8} k^{3} \sin \left[\frac{1}{2}\left(k^{*} z+k z^{*}\right)+\phi_{k}\right] .
\end{aligned}
$$

Hence,

$$
\tau=\left\langle H_{z z z} H_{z^{*} z^{*} z^{*}}\right\rangle=\left\langle\frac{1}{8}\left(k^{*}\right)^{3} \frac{1}{8} k^{3}\right\rangle=\frac{1}{64} K_{6} .
$$

Similarly, we have $\sigma=\frac{1}{16} K_{4}$.

\section{Probability distribution}

With the aid of the cumulants we can build the logarithm of the characteristic function [see Eq. (D3)], provided that we identify the appropriate variables in Fourier space. Consider $h_{z z}$ and $h_{z^{*} z^{*}}$, for example. These complex variables represent two real variables $\xi_{x}$ and $\xi_{y}$, the real and imaginary parts of $h_{z z}$. Let $\lambda_{x}$ and $\lambda_{y}$ be their Fourier counterparts. The characteristic function is

$$
\begin{aligned}
\chi\left(\lambda_{x}, \lambda_{y}, \ldots\right) & =\int d \xi_{x} d \xi_{y} \ldots p\left(\xi_{x}, \xi_{y}, \ldots\right) e^{i\left(\xi_{x} \lambda_{x}+\xi_{y} \lambda_{y}+\cdots\right)} \\
& =\left\langle e^{i\left(\xi_{x} \lambda_{x}+\xi_{y} \lambda_{y}+\cdots\right)}\right\rangle .
\end{aligned}
$$

The exponent can be written in terms of the complex variables

$$
\xi_{x} \lambda_{x}+\xi_{y} \lambda_{y}=h_{z z} \lambda_{z z}^{*}+h_{z^{*} z^{*}} \lambda_{z z}
$$

where we define $\lambda_{z z}=\frac{1}{2}\left(\lambda_{x}+i \lambda_{y}\right)$. Then, $\lambda_{z z}$ is the complex Fourier variable corresponding to $h_{z^{*} z^{*}}$ and we likewise introduce $\lambda_{z z z}$ and $\lambda_{z z z^{*}}$, which are conjugate to $h_{z^{*} z^{*} z^{*}}$ and $h_{z z^{*} z^{*}}$. We will define integrals with respect to the complex Fourier variables, e.g., with respect to $d^{2} h_{z z}$, as integrals over the real and imaginary parts of $h_{z z}$, and the inverse Fourier transform will be performed by integrating over the real and imaginary parts of the $\lambda$ 's.

The characteristic function is thus

$$
\begin{aligned}
\ln \chi= & -C_{2}\left(h_{z z}, h_{z^{*} z^{*}}\right) \lambda_{z^{*} z^{*}} \lambda_{z z}-C_{2}\left(h_{z z z}, h_{z^{*} z^{*} z^{*}}\right) \lambda_{z^{*} z^{*} z^{*}} \lambda_{z z z} \\
& -C_{2}\left(h_{z z z^{*}}, h_{z z^{*} z^{*}}\right) \lambda_{z z^{*} z^{*}} \lambda_{z z z^{*}} \\
& -i C_{3}\left(h_{z z}, h_{z z z^{*}}, h_{z^{*} z^{*} z^{*}}\right) \lambda_{z^{*} z^{*}} \lambda_{z z^{*} z^{*}} \lambda_{z z z} \\
& -i C_{3}\left(h_{z z}, h_{z z z^{*}}, h_{z^{*} z^{*} z^{*}}\right) \lambda_{z z} \lambda_{z z z^{*}} \lambda_{z^{*} z^{*} z^{*}} \\
& +\frac{1}{4} C_{4}\left(h_{z z z^{*}}, h_{z z z^{*}}, h_{z z^{*} z^{*}}, h_{z z^{*} z^{*}}\right) \lambda_{z z^{*} z^{*}}^{2} \lambda_{z z z^{*}}^{2} \\
& +\frac{1}{6} C_{4}\left(h_{z z z}, h_{z z^{*} z^{*}}, h_{z z^{*} z^{*}}, h_{z z^{*} z^{*}}\right) \lambda_{z^{*} z^{*} z^{*}} \lambda_{z z z^{*}}^{3} \\
& +\frac{1}{6} C_{4}\left(h_{z z z}, h_{z z^{*} z^{*}}, h_{z z^{*} z^{*}}, h_{z z^{*} z^{*}}\right) \lambda_{z z z} \lambda_{z z^{*} z^{*}}^{3}
\end{aligned}
$$

Upon entering the cumulants from Table I,

$$
\begin{aligned}
\ln \chi= & -\tilde{\sigma} \lambda_{z z} \lambda_{z^{*} z^{*}}-\tilde{\tau}\left(\lambda_{z z z} \lambda_{z^{*} z^{*} z^{*}}+\lambda_{z z z^{*}} \lambda_{z z^{*} z^{*}}\right) \\
& +3 i \sigma^{2}\left\langle f^{\prime \prime}(H)\right\rangle\left(\lambda_{z z} \lambda_{z z z^{*}} \lambda_{z^{*} z^{*} z^{*}}+\lambda_{z^{*} z^{*}} \lambda_{z z^{*} z^{*}} \lambda_{z z z}\right) \\
& -\sigma^{3}\left\langle f^{\prime \prime \prime}(H)\right\rangle\left(2 \lambda_{z z z^{*}}^{2} \lambda_{z z^{*} z^{*}}^{2}+\lambda_{z z z} \lambda_{z z^{*} z^{*}}^{3}+\lambda_{z^{*} z^{*} z^{*}} \lambda_{z z z^{*}}^{3}\right) .
\end{aligned}
$$

Here, $\tilde{\sigma}=\sigma\left(1+2\left\langle f^{\prime}(H)\right\rangle\right)$ and $\tilde{\tau}=\tau\left[1+2\left\langle f^{\prime}(H)\right\rangle\right]$ have been introduced. The factors in front of the cumulants are the factor $i^{k} / k$ ! in Eq. (D3) multiplied with the number of permutations of the $\lambda$ 's.

To obtain the probability distribution, we take the exponential and perform the inverse Fourier transformation [see Eq. (D1)]. This gives an integral over the exponential of a polynomial of degree 4 . However, all terms of degree 3 and 4 are of order $f$, so we can expand the exponential and be left with only square terms in the exponent. The result is

$$
\begin{aligned}
\chi= & \exp \left[\tilde{\sigma} \lambda_{z z} \lambda_{z^{*} z^{*}}-\tilde{\tau}\left(\lambda_{z z z} \lambda_{z^{*} z^{*} z^{*}}+\lambda_{z z z^{*}} \lambda_{z z^{*} z^{*}}\right)\right] \\
& \times\left(1+3 i \sigma^{2}\left\langle f^{\prime \prime}(H)\right\rangle\left(\lambda_{z z^{\prime}} \lambda_{z z z^{*}} \lambda_{z^{*} z^{*} z^{*}}+\lambda_{z^{*} z^{*}} \lambda_{z z^{*} z^{*}} \lambda_{z z z}\right)\right. \\
& \left.-\sigma^{3}\left\langle f^{\prime \prime \prime}(H)\right\rangle\left(2 \lambda_{z z z^{*}}^{2} \lambda_{z z^{*} z^{*}}^{2}+\lambda_{z z z} \lambda_{z z^{*} z^{*}}^{3}+\lambda_{z^{*} z^{*} z^{*}} \lambda_{z z z^{*}}^{3}\right)\right) .
\end{aligned}
$$

Now we can take the inverse Fourier transform. Note that $\lambda_{z z}$ and $\lambda_{z^{*} z^{*}}$ are each other's conjugate. Upon integrating the real and imaginary parts of $\lambda_{z z}$ and imposing $\lambda_{z^{*} z^{*}}=\lambda_{z z}^{*}$ (the same procedure applies to the other two pairs of $\lambda$ 's), one 
obtains

$$
\begin{aligned}
& p\left(h_{z z}, h_{z^{*} z^{*}}, h_{z z z}, h_{z^{*} z^{*} z^{*}}, h_{z z z^{*}}, h_{z z^{*} z^{*}}\right) \\
& =\int \frac{d^{2} \lambda_{z z} d^{2} \lambda_{z z z} d^{2} \lambda_{z z z^{*}}}{\pi^{6}} \chi \\
& \quad \times e^{-i\left(\lambda_{z z} h_{z z}+\lambda_{z z z} h_{z z z}+\lambda_{z z z^{*}} h_{z z z^{*}}+\text { conj. }\right)} .
\end{aligned}
$$

Note that the denominator is $\pi^{6}$ rather than $(2 \pi)^{6}$ because of the factor of $\frac{1}{2}$ in the definitions of the $\lambda$ 's [see Eq. (D27)].

The Fourier transform of a Gaussian function multiplied with a polynomial is easy to perform by noting that multiplying by $\lambda$ in Fourier space is equivalent to taking a derivative in normal space:

$$
\int d \lambda \lambda^{n} f(\lambda) e^{-i \lambda h}=\left(i \frac{\partial}{\partial h}\right)^{n} \int d \lambda f(\lambda) e^{-i \lambda h} .
$$

The inverse Fourier transform of the Gaussian part of the characteristic function is

$$
\begin{aligned}
& \int \frac{d^{2} \lambda_{z z} d^{2} \lambda_{z z z} d^{2} \lambda_{z z z^{*}}}{\pi^{6}} e^{-\tilde{\sigma}\left|\lambda_{z z}\right|^{2}-\tilde{\tau}\left(\left|\lambda_{z z z}\right|^{2}+\left|\lambda_{z z z^{*}}\right|^{2}\right)} e^{-i(\ldots)} \\
& =\frac{1}{\pi^{3} \tilde{\sigma} \tilde{\tau}^{2}} \exp \left(-\frac{1}{\tilde{\sigma}}\left|h_{z z}\right|^{2}-\frac{1}{\tilde{\tau}}\left(\left|h_{z z z}\right|^{2}+\left|h_{z z z^{*}}\right|^{2}\right)\right),
\end{aligned}
$$

and the final result reads as

$$
\begin{aligned}
p\left(h_{z z}, h_{z^{*} z^{*}}, h_{z z z}, h_{z^{*} z^{*} z^{*}}, h_{z z z^{*}}, h_{z z^{*} z^{*}}\right) & \\
= & {\left[1-3 \sigma^{2}\left\langle f^{\prime \prime}(H)\right\rangle \frac{1}{\tilde{\sigma} \tilde{\tau}^{2}}\left[2 \operatorname{Re}\left(h_{z^{*} z^{*}} h_{z z^{*} z^{*}} h_{z z z}\right)\right]\right.} \\
& -\sigma^{3}\left\langle f^{\prime \prime \prime}(H)\right\rangle\left(\frac{4}{\tilde{\tau}^{2}}-\frac{8\left|h_{z z z^{*}}\right|^{2}}{\tilde{\tau}^{3}}\right. \\
& \left.\left.+\frac{2\left|h_{z z z^{*}}\right|^{4}+2 \operatorname{Re}\left(h_{z z z} h_{z z^{*} z^{*}}^{3}\right)}{\tilde{\tau}^{4}}\right)\right] \\
& \times \frac{1}{\pi^{3} \tilde{\sigma} \tilde{\tau}^{2}} \exp \left(-\frac{\left|h_{z z}\right|^{2}}{\tilde{\sigma}}-\frac{\left|h_{z z z}\right|^{2}+\left|h_{z z z^{*}}\right|^{2}}{\tilde{\tau}}\right) .
\end{aligned}
$$

\section{Monstar fraction}

Once the joint probability distribution of the relevant derivatives is obtained, we can set $h_{z z}=h_{z^{*} z^{*}}=0$, which defines an umbilical point. The joint probability distribution states how likely it is that $h_{z z}$ and $h_{z^{*} z^{*}}$ are close to zero for a certain point $\vec{r}$. What we need, however, is for $h_{z z}$ and $h_{z^{*} z^{*}}$ to be exactly zero for a point close to $\vec{r}$ since we are looking for a density with respect to the $(x, y)$ plane. For this, we need to go from a probability density with respect to $h_{z z}$ and $h_{z^{*} z^{*}}$ to one with respect to $z$ and $z^{*}$. This is accomplished by multiplying $p$ with the Jacobian

$$
J=\left|\frac{\partial\left(h_{z z}, h_{z^{*} z^{*}}\right)}{\partial\left(z, z^{*}\right)}\right|=\left.|| h_{z z z}\right|^{2}-\left|h_{z z z^{*}}\right|^{2} \mid .
$$

The last step is to integrate this product over $h_{z z z}$ and $h_{z z z^{*}}$, either over all possible values, or just over those satisfying Eq. (D10), to get the density of umbilical points and the density of monstars, respectively:

$$
n=\int_{R} d^{2} h_{z z z} d^{2} h_{z z z^{*}} p\left(h_{z z}=0, h_{z z z}, h_{z z z^{*}}\right) J\left(h_{z z z}, h_{z z z^{*}}\right),
$$

where $R$ represents the range of integration: the entire space to get the density of all umbilical points, or Eq. (D10) for just the monstars.

First, we simplify by introducing polar coordinates

$$
h_{z z z}=\left|h_{z z z}\right| e^{i \phi}, \quad h_{z z z^{*}}=\left|h_{z z z^{*}}\right| e^{i \theta} .
$$

Next, we introduce

$$
u \equiv \frac{\left|h_{z z z}\right|}{\left|h_{z z z^{*}}\right|}, \quad \delta \equiv \frac{3 \theta-\phi}{2} .
$$

We find that we can rewrite the two conditions for monstars [Eq. (D10)] in terms of $u$ and $\delta$ only: the first one is simply $u>1$, while the other is

$$
\begin{aligned}
0 & <27-u^{4}-18 u^{2}-8 u^{3} \cos 2 \delta \\
& =(3-u)^{3}(1+u)-16 u^{3} \cos ^{2} \delta \\
& \Leftrightarrow \cos ^{2} \delta<\frac{(3-u)^{3}(1+u)}{16 u^{3}} .
\end{aligned}
$$

Since the fraction on the right-hand side is negative for $u>3$, we can extend the first condition to $1<u<3$.

The fact that the monstar conditions depend only on $u$ and $\delta$ can be understood as follows: the type of umbilic should not be affected by rescaling and/or rotating the plane. Rescaling would add the same (real) factor to $h_{z z z}$ and $h_{z z z^{*}}$, hence the type of umbilic should, as far as the moduli are concerned, depend only on the ratio $\left|h_{z z z}\right| /\left|h_{z z z^{*}}\right|$. A rotation introduces phase factors as given by Eq. (D11). We see that a rotation over an angle $\alpha$ causes $h_{z z z}$ to pick up a factor $e^{-3 i \alpha}$ while $h_{z z z^{*}}$ picks up $e^{-i \alpha}$. Therefore, the only combination of $\phi$ and $\theta$ that is invariant under rotations is $3 \theta-\phi$.

Now, we return our attention to the probability distribution. First, we rescale $h_{z z z}$ and $h_{z z z^{*}}$ :

$$
v \equiv \frac{h_{z z z}}{\sqrt{\tilde{\tau}}}, \quad w \equiv \frac{h_{z z z^{*}}}{\sqrt{\tilde{\tau}}} .
$$

This leads to

$$
\begin{aligned}
p\left(h_{z z}\right. & =0, v, w) J \\
\propto & \left(1-\frac{\sigma^{3}}{\tilde{\tau}^{2}}\left\langle f^{\prime \prime \prime}(H)\right\rangle\left(4-8|w|^{2}+2|w|^{4}+v w^{* 3}+v^{*} w^{3}\right)\right) \\
& \times\left. e^{-|v|^{2}-|w|^{2}}|| v\right|^{2}-|w|^{2} \mid .
\end{aligned}
$$

Here, we dropped an overall coefficient, which is of no importance since we are only interested in the ratio of the densities of monstars and all umbilical points. Note that $\tilde{\tau}$ now only appears in the term proportional to $f$. Since we are not interested in higher orders of $f$, we need only consider the leading order of $\tilde{\tau}$, which is $\tau$. For convenience, let us define

$$
\tilde{\varepsilon} \equiv \frac{\sigma^{3}}{\tau^{2}}\left\langle f^{\prime \prime \prime}(H)\right\rangle .
$$

Furthermore, note that multiplying $p$ with the constant $1+4 \tilde{\varepsilon}$, which we may do since we are only interested in the density 
of the ratios, causes the 4 inside the parentheses to be canceled out (up to first order).

Next, we move to polar coordinates, as we did before [31]:

$$
v \equiv \rho e^{i \phi}, \quad w \equiv r e^{i \theta}
$$

and then substitute $r=u \rho$ and $\phi=3 \theta-2 \delta$. With these transformations we have

$$
\begin{aligned}
n \propto & \int_{R} \rho^{3} u d \rho d u d \theta d \delta e^{-\rho^{2}\left(u^{2}+1\right)} \rho^{2}\left|u^{2}-1\right| \\
& \times\left(1-\tilde{\varepsilon}\left[-8 \rho^{2} u^{2}+2 \rho^{4}\left(u^{4}+u^{3} \cos 2 \delta\right)\right]\right) .
\end{aligned}
$$

Finally, we integrate over $\rho$ and $\theta$ to find the probability distribution $p(u, \delta)$. The integration over $\theta \operatorname{simply}$ gives a factor of $2 \pi$, while the integral over $\rho$ has the form of a polynomial times a Gaussian. For this we can use

$$
\int_{0}^{\infty} d \rho \rho^{2 n+1} e^{-\rho^{2}\left(u^{2}+1\right)}=\frac{n !}{2\left(u^{2}+1\right)^{n+1}} .
$$

The result is

$$
p(u, \delta) \propto \frac{u\left|u^{2}-1\right|}{\left(u^{2}+1\right)^{3}}\left(1+24 \tilde{\varepsilon} \frac{u^{2}(1-u \cos 2 \delta)}{\left(u^{2}+1\right)^{2}}\right) .
$$

The monstar density is proportional to the integral of $p(u, \delta)$ over the range

$$
1<u<3, \quad \cos ^{-1}\left(\sqrt{\frac{(3-u)^{3}(1+u)}{16 u^{3}}}\right)<\delta<\frac{1}{2} \pi,
$$

while the total density of umbilical points is proportional (with the same prefactor) to the integral over the range $0<u<\infty$, $0<\delta<\frac{1}{2} \pi$ (extending the integration range of $\delta$ from $\frac{1}{2} \pi$ to $2 \pi$ would just add a factor of 4 to both integrals). The latter can be done analytically: the integration over $\delta$ is trivial, while the remaining integral over $\rho$ can be split into two parts which, apart from a factor $\operatorname{sgn}\left(u^{2}-1\right)$, are both of the form

$$
\int d u \frac{u^{2}-1}{\left(u^{2}+1\right)^{2}}\left(\frac{u}{u^{2}+1}\right)^{n}=-\frac{1}{n+1}\left(\frac{u}{u^{2}+1}\right)^{n+1} .
$$

With this, we find

$$
\begin{aligned}
n_{\mathrm{tot}} \propto & \frac{1}{2} \pi\left[\frac{1}{2} \frac{u^{2}}{\left(u^{2}+1\right)^{2}}+24 \tilde{\varepsilon} \frac{1}{4} \frac{u^{4}}{\left(u^{2}+1\right)^{4}}\right]_{u=0}^{1} \\
& +\frac{1}{2} \pi\left[-\frac{1}{2} \frac{u^{2}}{\left(u^{2}+1\right)^{2}}-24 \tilde{\varepsilon} \frac{1}{4} \frac{u^{4}}{\left(u^{2}+1\right)^{4}}\right]_{u=1}^{\infty} \\
= & \frac{1}{8} \pi(1+3 \tilde{\varepsilon}) .
\end{aligned}
$$

For the monstar range, the integral over $\delta$ can be performed. The integral over the cosine gives

$$
\int_{\cos ^{-1}(\ldots)}^{\frac{1}{2} \pi} d \delta \cos 2 \delta=-\frac{1}{16 u^{3}} \sqrt{\left(u^{2}-1\right)\left(9-u^{2}\right)^{3}} .
$$

All together,

$$
\begin{aligned}
n_{\mathrm{M}} \propto & \int_{1}^{3} d u \frac{u\left(u^{2}-1\right)}{\left(u^{2}+1\right)^{3}} \sin ^{-1}\left(\sqrt{\frac{(3-u)^{3}(1+u)}{16 u^{3}}}\right) \\
& +\tilde{\varepsilon} \int_{1}^{3} d u \frac{u\left(u^{2}-1\right)}{\left(u^{2}+1\right)^{3}} \\
& \times\left[\frac{24 u^{2}}{\left(u^{2}+1\right)^{2}} \sin ^{-1}\left(\sqrt{\frac{(3-u)^{3}(1+u)}{16 u^{3}}}\right)\right. \\
& \left.+\frac{3 \sqrt{\left(u^{2}-1\right)\left(9-u^{2}\right)^{3}}}{2\left(u^{2}+1\right)^{2}}\right] \\
\equiv & I_{1}+\tilde{\varepsilon} I_{2} .
\end{aligned}
$$

The integrals can be done numerically. The monstar fraction is then

$$
\begin{aligned}
\alpha_{M} & =\frac{n_{\mathrm{M}}}{n_{\mathrm{tot}}}=\frac{I_{1}+\tilde{\varepsilon} I_{2}}{\frac{1}{8} \pi(1+3 \tilde{\varepsilon})}=\frac{8}{\pi}\left[I_{1}+\tilde{\varepsilon}\left(I_{2}-3 I_{1}\right)\right]+O\left(\tilde{\varepsilon}^{2}\right) \\
& =0.053+0.429 \mu\left\langle f^{\prime \prime \prime}(H)\right\rangle+O\left(f^{2}\right),
\end{aligned}
$$

where

$$
\mu \equiv \frac{\sigma^{3}}{\tau^{2}}=\frac{K_{4}^{3}}{K_{6}^{2}} \quad(0 \leqslant \mu \leqslant 1) .
$$

Note that the zeroth order result matches the one in [7]. Remember that we set $K_{0}=\left\langle H^{2}\right\rangle=1$ for convenience; if we drop this condition, then $K_{0}$ enters the denominator of the expression above.

By comparing the fraction of monstars in a given field to the formula just found, we can determine one parameter of the deviation from a Gaussian distribution $\left\langle f^{\prime \prime \prime}(H)\right\rangle$. This assumes that the field $h$ is given by $h=H+f(H)$. To test this, one could, if possible, also measure the distribution $p(u, \delta)$ to test that it has the right form [Eq. (D45)]. Measuring $p(\delta)$ only could also suffice. For a Gaussian field $H$, all values of $\delta$ should be equally likely, whereas integrating Eq. (D45) shows that the distribution we expect for $h$ is

$$
p(\delta)=\frac{1}{\pi}(1-4 \tilde{\varepsilon} \cos 2 \delta),
$$

where we define $\delta$ to lie between $-\pi / 2$ and $\pi / 2$.

\section{APPENDIX E: PROOF OF EQ. (D16)}

In this section, we prove the identity

$$
\begin{aligned}
& C_{n}\left(f\left(H_{1}\right), H_{2}, \ldots, H_{n}\right) \\
& \quad=\left\langle f^{(n-1)}\left(H_{1}\right)\right\rangle\left\langle H_{1} H_{2}\right\rangle\left\langle H_{1} H_{3}\right\rangle \ldots\left\langle H_{1} H_{n}\right\rangle
\end{aligned}
$$

for Gaussian variables $H_{i}$.

Recall the definition of a cumulant [Eq. (D3)]. The characteristic function $\chi$ is the Fourier transform of the probability distribution [see Eq. (D1)], which for Gaussian variables is Eq. (12). This leads to the identity

$$
\begin{aligned}
& C_{n}(\left.f\left(H_{1}\right), H_{2}, \ldots, H_{n}\right) \\
&=(-i)^{n} \frac{\partial}{\partial \lambda_{1}} \ldots \frac{\partial}{\partial \lambda_{n}} \ln \int d h_{1} \ldots d h_{n} e^{i\left[\lambda_{1} f\left(h_{1}\right)+\lambda_{2} h_{2}+\cdots+\lambda_{n} h_{n}\right]} \\
& \quad \times\left.\frac{\exp \left(-\frac{1}{2} \sum_{i j} \sigma_{i j}^{-1} h_{i} h_{j}\right)}{(2 \pi)^{n / 2} \sqrt{\operatorname{det} \sigma}}\right|_{\lambda_{1}=\cdots=\lambda_{n}=0} .
\end{aligned}
$$


First, the integration over $h_{2}$ through $h_{n}$ is performed. This partial Fourier transform is not trivial. If $h_{1}$ were included, the answer would be simply Eq. (D6). We can, however, use this result and take the inverse Fourier transform of it with respect to $\lambda_{1}$ to get the desired result:

$$
\begin{aligned}
\int & d h_{2} \ldots d h_{n} e^{i\left(\lambda_{2} h_{2}+\cdots+\lambda_{n} h_{n}\right)} \frac{\exp \left(-\frac{1}{2} \sum_{i j} \sigma_{i j}^{-1} h_{i} h_{j}\right)}{(2 \pi)^{n / 2} \sqrt{\operatorname{det} \sigma}} \\
= & \int \frac{d \lambda_{1}}{2 \pi} e^{-i \lambda_{1} h_{1}} \exp \left(-\frac{1}{2} \sum_{i j} \sigma_{i j} \lambda_{i} \lambda_{j}\right) \\
= & \int \frac{d \lambda_{1}}{2 \pi} \exp \left[-\frac{1}{2} \sigma_{11} \lambda_{1}^{2}-\left(i h_{1}+\sum_{j \geqslant 2} \sigma_{1 j} \lambda_{j}\right) \lambda_{1}\right. \\
& \left.-\frac{1}{2} \sum_{i, j \geqslant 2} \sigma_{i j} \lambda_{i} \lambda_{j}\right] \\
= & \frac{1}{\sqrt{2 \pi \sigma_{11}}} \exp \left[\frac{1}{2 \sigma_{11}}\left(i h_{1}+\sum_{j \geqslant 2} \sigma_{1 j} \lambda_{j}\right)^{2}\right. \\
& \left.-\frac{1}{2} \sum_{i, j \geqslant 2} \sigma_{i j} \lambda_{i} \lambda_{j}\right] .
\end{aligned}
$$

The integration was performed by completing the square. Now, we do the Fourier transform with respect to $h_{1}$ and include the logarithm present in Eq. (E2), which leads to

$$
\begin{aligned}
\ln \int & d h_{1} \ldots d h_{n} e^{i\left[\lambda_{1} f\left(h_{1}\right)+\lambda_{2} h_{2}+\cdots+\lambda_{n} h_{n}\right]} \\
\times & \frac{\exp \left(-\frac{1}{2} \sum_{i j} \sigma_{i j}^{-1} h_{i} h_{j}\right)}{(2 \pi)^{n / 2} \sqrt{\operatorname{det} \sigma}} \\
= & \ln \int d h_{1} e^{i \lambda_{1} f\left(h_{1}\right)} \frac{1}{\sqrt{2 \pi \sigma_{11}}} \\
& \times e^{\frac{1}{2 \sigma_{11}}\left[i h_{1}+\sum_{j \geqslant 2} \sigma_{1 j} \lambda_{j}\right]^{2}-\frac{1}{2} \sum_{i, j \geqslant 2} \sigma_{i j} \lambda_{i} \lambda_{j}} \\
= & -\frac{1}{2} \sum_{i, j \geqslant 2} \sigma_{i j} \lambda_{i} \lambda_{j}+\ln \int d h_{1} e^{i \lambda_{1} f\left(h_{1}\right)} \frac{1}{\sqrt{2 \pi \sigma_{11}}} \\
& \times e^{\frac{1}{2 \sigma_{11}}\left[i h_{1}+\sum_{j \geqslant 2} \sigma_{1 j} \lambda_{j}\right]^{2}} .
\end{aligned}
$$

In accordance with Eq. (E2), we must take the derivative of this equation with respect to the $\lambda$ 's and set them to zero. First, the derivative with respect to $\lambda_{1}$ is taken. This causes the first term to vanish since it does not depend on $\lambda_{1}$. This simplification can be regarded as the main reason why the final result depends on $f$ in a rather simple way. What remains is

$$
\begin{gathered}
-\left.i \frac{\partial}{\partial \lambda_{1}} \ln \int d h_{1} e^{i \lambda_{1} f\left(h_{1}\right)} \frac{1}{\sqrt{2 \pi \sigma_{11}}} e^{\frac{1}{2 \sigma_{11}}\left[i h_{1}+\sum_{j \geqslant 2} \sigma_{1 j} \lambda_{j}\right]^{2}}\right|_{\lambda_{1}=0} \\
=\frac{\int d h_{1} f\left(h_{1}\right) \exp \left[\frac{1}{2 \sigma_{11}}\left(i h_{1}+\sum_{j \geqslant 2} \sigma_{1 j} \lambda_{j}\right)^{2}\right]}{\int d h_{1} \exp \left[\frac{1}{2 \sigma_{11}}\left(i h_{1}+\sum_{j \geqslant 2} \sigma_{1 j} \lambda_{j}\right)^{2}\right]} \\
=\frac{1}{\sqrt{2 \pi \sigma_{11}}} \int d h_{1} f\left(h_{1}\right) e^{\frac{1}{2 \sigma_{11}}\left[i h_{1}+\sum_{j \geqslant 2} \sigma_{1 j} \lambda_{j}\right]^{2}}
\end{gathered}
$$

For each $\lambda_{k}$ with $k \geqslant 2$ the derivative yields

$$
\begin{aligned}
& -i \frac{\partial}{\partial \lambda_{k}} e^{\frac{1}{2 \sigma_{11}}\left[i h_{1}+\sum_{j \geqslant 2} \sigma_{1 j} \lambda_{j}\right]^{2}} \\
& =-i \frac{\sigma_{1 k}}{\sigma_{11}}\left(i h_{1}+\sum_{j \geqslant 2} \sigma_{1 j} \lambda_{j}\right) e^{\frac{1}{2 \sigma_{11}}\left[i h_{1}+\sum_{j \geqslant 2} \sigma_{1 j} \lambda_{j}\right]^{2}} .
\end{aligned}
$$

Applying this for all $k$ and subsequently setting all $\lambda_{k}$ to zero then gives

$$
\begin{gathered}
(-i)^{n-1} \frac{\partial}{\partial \lambda_{2}} \ldots \frac{\partial}{\partial \lambda_{n}} e^{\frac{1}{2 \sigma_{11}}\left[i h_{1}+\sum_{j \geqslant 2} \sigma_{1 j} \lambda_{j}\right]^{2}} \\
=\left(\frac{h_{1}}{\sigma_{11}}\right)^{n-1}\left(\prod_{j \geqslant 2} \sigma_{1 j}\right) e^{-h_{1}^{2} /\left(2 \sigma_{11}\right)} .
\end{gathered}
$$

This results in

$$
\begin{aligned}
C_{n} & \left(f\left(H_{1}\right), H_{2}, \ldots, H_{n}\right) \\
= & \left(\prod_{j \geqslant 2} \sigma_{1 j}\right) \frac{1}{\sqrt{2 \pi \sigma_{11}}} \int d h_{1} f\left(h_{1}\right)\left(\frac{h_{1}}{\sigma_{11}}\right)^{n-1} e^{-h_{1}^{2} /\left(2 \sigma_{11}\right)} .
\end{aligned}
$$

Integrating by parts $n-1$ times leads to

$$
\begin{aligned}
C_{n} & \left(f\left(H_{1}\right), H_{2}, \ldots, H_{n}\right) \\
= & \left(\prod_{j \geqslant 2} \sigma_{1 j}\right) \frac{1}{\sqrt{2 \pi \sigma_{11}}} \int d h_{1} f^{(n-1)}\left(h_{1}\right) e^{-h_{1}^{2} /\left(2 \sigma_{11}\right)} .
\end{aligned}
$$

Finally, we identify the integral (along with the prefactor) as the expectation value of $f^{(n-1)}$ and $\sigma_{1 j}=\left\langle H_{1} H_{j}\right\rangle$, which gives us

$$
\begin{aligned}
& C_{n}\left(f\left(H_{1}\right), H_{2}, \ldots, H_{n}\right) \\
& \quad=\left\langle f^{(n-1)}\left(H_{1}\right)\right\rangle\left\langle H_{1} H_{2}\right\rangle\left\langle H_{1} H_{3}\right\rangle \ldots\left\langle H_{1} H_{n}\right\rangle,
\end{aligned}
$$

the equation we set out to prove.
[1] S. Dodelson, Modern Cosmology (Academic, Amsterdam, 2003).

[2] M. Kardar, G. Parisi, and Y. C. Zhang, Phys. Rev. Lett. 56, 889 (1986).

[3] K. J. Worsley, S. Marrett, P. Neelin, A. C. Vandal, K. J. Friston, and A. C. Evans, Human Brain Mapping 4, 58 (1996).

[4] F. Flossmann, K. O'Holleran, M. R. Dennis, and M. J. Padgett, Phys. Rev. Lett. 100, 203902 (2008).
[5] A. Weinrib and B. I. Halperin, Phys. Rev. B 26, 1362 (1982).

[6] M. S. Longuet-Higgins, Philos. Trans. R. Soc., A 250, 157 (1957).

[7] M. V. Berry and J. H. Hannay, J. Phys. A: Math. Gen. 10, 1809 (1977).

[8] G. Foltin, J. Phys. A: Math. Gen. 36, 1729 (2003).

[9] G. Foltin, J. Phys. A: Math. Gen. 36, 4561 (2003).

[10] I. Freund and M. Wilkinson, J. Opt. Soc. Am. A 15, 2892 (1998). 
[11] T. H. Beuman, A. M. Turner, and V. Vitelli, Proc. Natl. Acad. Sci. USA 109, 19943 (2012).

[12] T. H. Beuman, A. M. Turner, and V. Vitelli, Phys. Rev. E 87, 022142 (2013).

[13] R. Kamien, Rev. Mod. Phys. 74, 953 (2002).

[14] M. R. Dennis, J. Phys. A: Math. Gen. 36, 6611 (2003).

[15] M. Longuet-Higgins, Philos. Trans. R. Soc., A 249, 321 (1957).

[16] M. R. Dennis, Opt. Lett. 33, 2572 (2008).

[17] A. F. Heavens and R. K. Sheth, Mon. Not. R. Astron. Soc. 310, 1062 (1999).

[18] S. Gupta and A. F. Heavens, AIP Conf. Proc. 555, 337 (2001).

[19] H. Hoekstra and B. Jain, Ann. Rev. Nucl. Part. Sci. 58, 99 (2008).

[20] V. Vitelli, B. Jain, and R. D. Kamien, J. Cosmol. Astropart. Phys. 09, 034 (2009).

[21] M. R. Dennis and K. Land, Mon. Not. R. Astron. Soc. 383, 424 (2008).
[22] D. Huterer and T. Vachaspati, Phys. Rev. D 72, 043004 (2005).

[23] T. Vachaspati and A. Lue, Phys. Rev. D 67, 121302 (2003).

[24] P. D. Naselsky and D. I. Novikov, Astrophys. J. 507, 31 (1998).

[25] S. D. Hudson and E. L. Thomas, Phys. Rev. Lett. 62, 1993 (1989).

[26] I. E. Dzyaloshinskii, Zh. Eksp. Teor. Fiz. 58, 1443 (1970) [Sov. Phys.-JETP 31, 773 (1970)].

[27] B. I. Halperin, Physics of Defects, Les Houches XXXV NATO ASI (North Holland, Ansterdam, 1981).

[28] F. Liu and G. F. Mazenko, Phys. Rev. B 46, 5963 (1992).

[29] N. G. van Kampen, Stochastic Processes in Physics and Chemistry (North-Holland, Amsterdam, 1981).

[30] P. M. Chaikin and T. C. Lubensky, Principles of Condensed Matter Physics (Cambridge University Press, Cambridge, 2000).

[31] Apart from the sign of $\phi$ and a numerical factor, the variables $r$, $\rho, \phi$, and $\theta$ match their respective counterparts in [7]. 